TRANSACTIONS OF THE

AMERICAN MATHEMATICAL SOCIETY

Volume 352, Number 10, Pages 4603-4640

S 0002-9947(00)02541-1

Article electronically published on June 13, 2000

\title{
A GLOBAL APPROACH TO FULLY NONLINEAR PARABOLIC PROBLEMS
}

\author{
ATHANASSIOS G. KARTSATOS AND IGOR V. SKRYPNIK
}

Abstract. We consider the general initial-boundary value problem

$$
\begin{aligned}
& \text { (1) } \frac{\partial u}{\partial t}-F\left(x, t, u, \mathcal{D}^{1} u, \mathcal{D}^{2} u\right)=f(x, t), \quad(x, t) \in Q_{T} \equiv \Omega \times(0, T), \\
& \text { (2) } G\left(x, t, u, \mathcal{D}^{1} u\right)=g(x, t), \quad(x, t) \in S_{T} \equiv \partial \Omega \times(0, T), \\
& \text { (3) } u(x, 0)=h(x), \quad x \in \Omega,
\end{aligned}
$$

where $\Omega$ is a bounded open set in $\mathcal{R}^{n}$ with sufficiently smooth boundary. The problem (1)-(3) is first reduced to the analogous problem in the space $W_{p}^{(4), 0}\left(Q_{T}\right)$ with zero initial condition and

$$
f \in W_{p}^{(2), 0}\left(Q_{T}\right), g \in W_{p}^{\left(3-\frac{1}{p}\right), 0}\left(S_{T}\right) .
$$

The resulting problem is then reduced to the problem $A u=0$, where the operator $A: W_{p}^{(4), 0}\left(Q_{T}\right) \rightarrow\left[W_{p}^{(4), 0}\left(Q_{T}\right)\right]^{*}$ satisfies Condition $(S)_{+}$. This reduction is based on a priori estimates which are developed herein for linear parabolic operators with coefficients in Sobolev spaces. The local and global solvability of the operator equation $A u=0$ are achieved via topological methods developed by I. V. Skrypnik. Further applications are also given involving relevant coercive problems, as well as Galerkin approximations.

\section{INTRODUCTION AND PRELIMINARIES}

This paper is devoted to the reduction of fully nonlinear parabolic problems to operator equations involving operators satisfying Condition $(S)_{+}$. This process enables us to employ topological methods based on Skrypnik's degree theory in [14. For simplicity, we consider only second order parabolic equations. However, an analogous approach can be developed for nonlinear parabolic equations of higher order with general nonlinear boundary operators satisfying Lopatynskij's condition.

In what follows, the symbols $\mathcal{R}, \mathcal{R}_{+}$denote the real line and the set $[0, \infty)$, respectively. The symbols $\partial D, \bar{D}$ denote the strong boundary and the strong closure of the set $D$, respectively, in a Banach space. We use the notation $B(x, r)$ to denote the open ball around the point $x$ with radius $r>0$. The symbols " $\rightarrow$ " " $\rightarrow$ ") denote strong (weak) convergence. Unless otherwise specified, the term "continuous", for a mapping $f$ acting between two real Banach spaces, means that $f$ is strongly continuous.

Received by the editors April 18, 1997 and, in revised form, May 7, 1998.

1991 Mathematics Subject Classification. Primary 35K55; Secondary 35K30, 35K35.

Key words and phrases. Initial-boundary value problem, mapping of type $(S)_{+}$, Skrypnik's degree theory for demicontinuous mappings of type $(S)_{+}$, Galerkin approximation.

This research was partially supported by an NSF-NRC COBASE grant. 
Let $\Omega$ denote a bounded open set in $\mathcal{R}^{n}$ with sufficiently smooth boundary. We consider initial-boundary value problems of the type

$$
\begin{gathered}
\frac{\partial u}{\partial t}-F\left(x, t, u, \mathcal{D}^{1} u, \mathcal{D}^{2} u\right)=f(x, t), \quad(x, t) \in Q_{T} \equiv \Omega \times(0, T), \\
G\left(x, t, u, \mathcal{D}^{1} u\right)=g(x, t), \quad(x, t) \in S_{T} \equiv \partial \Omega \times(0, T), \\
u(x, 0)=h(x), \quad x \in \Omega,
\end{gathered}
$$

in the space $W_{p}^{(4)}\left(Q_{T}\right)=W_{p}^{4,2}\left(Q_{T}\right)$ for $p>\frac{n+2}{2}, p \neq 3$.

We assume that $F(x, t, \xi),(x, t, \xi) \in \overline{Q_{T}} \times \mathcal{R}^{\mathcal{N}}\left(\mathcal{N} \equiv 1+n+\frac{n(n+1)}{2}\right)$, and $G(y, t, \zeta),(y, t, \zeta) \in \overline{S_{T}} \times \mathcal{R}^{n+1}$, are sufficiently smooth functions. We also assume that the following inequalities hold:

$$
\begin{gathered}
\sum_{|\alpha|=2} F_{\alpha}(x, t, \xi) \eta^{\alpha} \geq \nu(|\xi|)|\eta|^{2}, \quad(x, t, \xi) \in \overline{Q_{T}} \times \mathcal{R}^{\mathcal{N}}, \eta \in \mathcal{R}^{n}, \\
\sum_{|\alpha|=1} G_{\alpha}(y, t, \zeta) n_{\alpha}(y) \geq \nu(|\zeta|), \quad(y, t, \zeta) \in \overline{S_{T}} \times \mathcal{R}^{n+1}
\end{gathered}
$$

Here, $F_{\alpha}(x, t, \xi) \equiv \frac{\partial}{\partial \xi_{\alpha}} F(x, t, \xi), G_{\alpha}(y, t, \zeta)=\frac{\partial}{\partial \zeta_{\alpha}} G(y, t, \zeta), n_{\alpha}$ is the unit vector in the direction of the outward normal to $\partial \Omega$ at the point $(y, t) \in \overline{S_{T}}$, and $\nu: \overline{\mathcal{R}_{+}} \rightarrow$ $\mathcal{R}_{+}$is some continuous function. We have not imposed any growth restriction on the behavior of the functions $F(x, t, \xi), G(y, t, \zeta)$ with respect to $\xi$ and $\zeta$.

The functions on the right-hand sides of (1.1)-(1.3) satisfy the inclusions

$$
f \in W_{p}^{(2)}\left(Q_{T}\right), \quad g \in W_{p}^{\left(3-\frac{1}{p}\right)}\left(S_{T}\right), \quad h \in W_{p}^{4-\frac{2}{p}}(\Omega),
$$

as well as some compatibility conditions for $x \in \partial \Omega, t=0$. Note that from our restriction on $p$ and Sobolev embeddings (see Lemma 2.2 below) it follows that $f(x, t), g(x, t), h(x)$ and the solution $u(x, t)$ belong to Hölder functions spaces. In particular, $f \in \mathcal{C}^{\delta, \delta / 2}\left(\overline{Q_{T}}\right)$ and $u \in \mathcal{C}^{2+\delta, 1+\delta / 2}\left(\overline{Q_{T}}\right)$, where $0<\delta<2-(n+2) / p$.

In a standard way, it is possible to reduce the problem (1.1)-(1.3) to the analogous problem in the space $W^{(4), 0}\left(Q_{T}\right)$ with zero initial condition and $f \in W_{p}^{(2), 0}\left(Q_{T}\right)$, $g \in W_{p}^{\left(3-\frac{1}{p}\right), 0}\left(S_{T}\right)$. In the rest of this section we assume that this reduction has already taken place.

The main result of this paper consists of the reduction of the problem (1.1)-(1.3) with zero initial condition to the operator equation

$$
A u=0
$$

in the space $W_{p}^{(4), 0}\left(Q_{T}\right)$ with a bounded and continuous operator $A$ acting from the space $W_{p}^{(4), 0}\left(Q_{T}\right)$ into the dual space $\left[W_{p}^{(4), 0}\left(Q_{T}\right)\right]^{*}$. The operator $A$ satisfies "Condition $(S)_{+}$", which is defined as follows: for every sequence of functions $u_{j} \in W_{p}^{(4), 0}\left(Q_{T}\right)$, which converges weakly to some function $u_{0}$ and is such that

$$
\limsup _{j \rightarrow \infty}\left\langle A u_{j}, u_{j}-u_{0}\right\rangle \leq 0
$$

we have that $u_{j}$ converges strongly to $u_{0}$.

We note that Condition $(S)_{+}$is Condition $\alpha$ in the monograph [13].

These properties of the operator $A$ allow us to study the solvability of the equation (1.7) via topological methods developed by Skrypnik in [13], [14]. Namely, 
Skrypnik created a topological degree function, $\operatorname{Deg}(A, \overline{\mathcal{D}}, 0)$, for such operators $A$, where $D$ is an open and bounded subset of $W_{p}^{(4), 0}\left(Q_{T}\right)$, provided that $0 \notin A(\partial \mathcal{D})$.

The reduction of the problem (1.1)-(1.3) to an operator equation is based upon a priori estimates for linear parabolic operators with coefficients from Sobolev spaces, obtained in this paper, which are analogous to well known a priori estimates for linear operators with smooth coefficients.

We also develop in this paper some applications of the topological approach. In particular, we show the local solvability of the problem (1.1)-(1.3), the conditional solvability of the same problem for an arbitrary time $T$, the solvability of the relevant coercive problem, and the strong convergence of the Galerkin approximants.

In the case of a fully nonlinear elliptic boundary value problem analogous topological methods were developed in the monograph [14].

The local existence of the Cauchy-Dirichlet problem for fully nonlinear parabolic equations was established by other methods in the papers of Hudjaev [4, Kruzhkov, Castro and Lopes [6], and Sopolov [16]. The problem of the existence in the large has been studied by Krylov [7, Lunardi 10] and Wang [18. The local approach for problems with nonlinear boundary conditions was developed by Amann [2] and Acquistapace and Terreni [1].

A priori estimates and the solvability of the problem (1.1)-(1.3) in the space $\mathcal{C}^{2+\lambda, 1+\lambda / 2}\left(\overline{Q_{T}}\right)$ with natural restrictions on the coefficients were established by Dong [3], Lieberman [9] and Uraltseva [17. The results of this paper involving the solvability in $W_{p}^{(4)}\left(Q_{T}\right)$ cannot be obtained from the results in [3], [9], 17] and well known a priori estimates of solutions of linear problems with smooth coefficients [8]. On the other hand, using our approach and results from [3], 9], [17], we can establish the solvability in $W_{p}^{(4)}\left(Q_{T}\right)$ of the problem (1.1)-(1.3) if we know a priori estimates in $\mathcal{C}\left(\overline{Q_{T}}\right)$ for the solutions of the corresponding parametric nonlinear problem.

This paper is organized in the following way. In Section 2 we formulate some auxiliary propositions for the function spaces considered herein. We also state the initial-boundary value problem and the corresponding problem with zero initial condition. In Section 3 we reduce the differential problem to the operator equation and formulate our main results. In Section 4 we derive a priori estimates for linear parabolic problems with coefficients from Sobolev spaces. The properties of the nonlinear operator $A$ are studied in Section 5. Some applications of the topological method to the study of the solvability of nonlinear parabolic problems are contained in Section 6. In Section 7 we establish the strong convergence of the Galerkin approximants for the parabolic problems under consideration.

\section{Statement of the PROBlem AND PRELIMINARIES}

1. Function spaces. We study below the initial-boundary value problem for nonlinear parabolic equations in the cylindrical domain $Q_{T} \equiv \Omega \times(0, T) \times \mathcal{R}^{n+1}$, where $T>0$ is a finite number and $\Omega$ is a bounded open set in $\mathcal{R}^{n}$ with smooth boundary. We now elaborate on our notations, definitions and auxiliary results.

We use well known Sobolev spaces which can be found in 8]. Namely, we use the spaces $W_{p}^{l, l / 2}\left(Q_{T}\right)$ for positive, non-integer, or even, numbers $l$, and the spaces $W_{p}^{l, l / 2}(\Omega), W_{p}^{l, l / 2}\left(S_{T}\right)$ for positive, non-integer $l$, which are defined in [8, pp. 5, 70, 81]. We assume that the boundary of the domain $\Omega$ belongs to the class $\mathcal{C}^{l_{0}}$, where 
$l_{0}=\max \{l, 1\}$. We also use the notation

$$
W_{p}^{(l)}\left(Q_{T}\right)=W_{p}^{l, \frac{l}{2}}\left(Q_{T}\right), \quad W_{p}^{(l)}\left(S_{T}\right)=W_{p}^{l, \frac{l}{2}}\left(S_{T}\right),
$$

and denote the norms in the spaces $L_{p}\left(Q_{T}\right), W_{p}^{(l)}\left(Q_{T}\right), W_{p}^{(l)}\left(S_{T}\right), W_{p}^{l}(\Omega)$ by $\|\cdot\|_{p, Q_{T}},\|\cdot\|_{p, Q_{T}}^{(l)},\|\cdot\|_{p, S_{T}}^{(l)},\|\cdot\|_{p, \Omega}^{(l)}$, respectively.

Analogounsly, we use the notation

$$
\mathcal{C}^{(l)}\left(\overline{Q_{T}}\right)=H^{l, \frac{l}{2}}\left(\overline{Q_{T}}\right), \quad \mathcal{C}^{(l)}\left(S_{T}\right)=H^{l, \frac{l}{2}}\left(S_{T}\right),
$$

for positive, non-integer $l$, and denote the norms in these spaces by $\|\cdot\|_{Q_{T}}^{(l)}$ and $\|\cdot\|_{S_{T}}^{(l)}$, respectively. The spaces $H^{l, l / 2}\left(\overline{Q_{T}}\right), H^{l, l / 2}\left(S_{T}\right)$ can be found in 8 pp. 7 , 81].

We use multi-index notation. Let $\alpha=\left(\alpha_{1}, \ldots, \alpha_{n}\right)$ be a multi-index with nonnegative integer components $\alpha_{i}$ and length $|\alpha|=\alpha_{1}+\ldots+\alpha_{n}$. For $\xi=\left(\xi_{1}, \ldots, \xi_{n}\right) \in$ $\mathcal{R}^{n}$ we let $\xi^{\alpha}=\xi_{1}^{\alpha_{1}} \cdots \xi_{n}^{\alpha_{n}}$. We also let

$$
\mathcal{D}^{\alpha} u(x, t)=\left(\frac{\partial}{\partial x_{1}}\right)^{\alpha_{1}} \cdots\left(\frac{\partial}{\partial x_{n}}\right)^{\alpha_{n}} u(x, t), \quad \mathcal{D}^{k} u(x, t)=\left\{\mathcal{D}^{\alpha} u(x, t):|\alpha|=k\right\} .
$$

The study of initial-boundary value problems for parabolic equations is based on properties of traces of functions from the spaces $W_{p}^{(l)}\left(Q_{T}\right)$ which are given in the following lemma.

Lemma 2.1. Assume that $l$ is a positive even number and $1<p<\infty$. Then, for an arbitrary function $u \in W_{p}^{(l)}\left(Q_{T}\right)$, we have

$$
\left\|\left(\frac{\partial}{\partial t}\right)^{s_{1}} \mathcal{D}^{\alpha} u\right\|_{p, \Omega_{0}}^{(k)} \leq C_{1}\|u\|_{p, Q_{T}}^{(l)}, \quad 2 s_{1}+|\alpha|<l-\frac{2}{p},
$$

and

$$
\left\|\left(\frac{\partial}{\partial t}\right)^{s_{2}} \mathcal{D}^{\beta} u\right\|_{p, S_{T}}^{(j)} \leq C_{2}\|u\|_{p, Q_{T}}^{(l)}, \quad 2 s_{2}+|\alpha|<l-\frac{1}{p},
$$

where $k=l-2 s_{1}-|\alpha|-\frac{2}{p}, j=l-2 s_{2}-|\beta|-\frac{1}{p}, \Omega_{0}=\Omega \times\{0\}$, and the constants $C_{1}, C_{2}$ are independent of $u$.

The proof of Lemma 2.1 can be found in [5].

We also need some interpolation estimates for domains of small diameters. We formulate these estimates only for the model domains considered herein.

Lemma 2.2. Assume that $l$ is either an even integer or a non-integer positive number, $1<p<\infty, G_{T}(r)=B(r) \times(0, T), B(r)=\left\{x \in \mathcal{R}^{n}:|x|<r\right\}, G_{T}^{(0)}(r)=$ $G_{T}(r) \cap\left\{x_{n}=0\right\}, G_{T}^{(1)}(r)=G_{T}(r)$, and let $u(x, t)$ be an arbitrary function in the space $W_{p}^{(l)}\left(G_{T}(r)\right)$. Then, for $i=0,1$, the following estimates hold with $0<h \leq$ $\min \{r, \sqrt{T}\}$ and constants $C_{3}, C_{4}, C_{5}$ independent of $u, h$.

(i) The inequality

$$
\left\|\left(\frac{\partial}{\partial t}\right)^{s} \mathcal{D}^{\alpha} u\right\|_{p, G_{T}^{(i)}(r)} \leq C_{3} h^{l \epsilon_{i}}\left\{|u|_{p, G_{T}(r)}^{(l)}+h^{-l}\|u\|_{p, G_{T}(r)}\right\}
$$


holds for all $s, \alpha, \epsilon_{i}$ satisfying the condition

$$
\epsilon_{i}=1-\frac{2 s+|\alpha|}{l}-a_{i}>0, \quad \text { where } a_{1}=0, a_{0}=\frac{1}{l p} .
$$

(ii) The inequality

$$
\left|\left(\frac{\partial}{\partial t}\right)^{s} \mathcal{D}^{\alpha} u\right|_{p, G_{T}^{(i)}(r)}^{\left(k_{i}\right)} \leq C_{4} h^{l \epsilon_{i}-k_{i}}\left\{|u|_{p, G_{T}(r)}^{(l)}+h^{-l}\|u\|_{p, G_{T}(r)}\right\}
$$

holds, where $s, \alpha, \epsilon_{i}$ satisfy the condition (2.6) and the non-integer numbers $k_{i}$ are such that $0<k_{0} \leq \epsilon_{0} l, 0<k_{1}<\epsilon_{1} l$.

(iii) The inequality

$$
|u|_{G_{T}(r)}^{(j)} \leq C_{5} h^{l-j-\frac{n+2}{p}}\left\{|u|_{p, G_{T}(r)}^{(l)}+h^{-l}\|u\|_{p, G_{T}(r)}\right\}
$$

holds, where $l p>n+2$ and the number $j$ is such that $0 \leq j<l-\frac{n+2}{p}$.

The assertions of Lemma 2.2 follow from the results in [5]. Another interpolation inequality is given by the the next lemma.

Lemma 2.3. Let $l$ be a positive integer and $1<p<\infty$. There exists a constant $C_{6}$, independent of $u$ and $T$, such that for any $u \in W_{p}^{(l)}\left(Q_{T}\right) \cap L_{\infty}\left(Q_{T}\right)$ the inequality

$$
\sum_{|\alpha|=j}\left\|\mathcal{D}^{\alpha} u\right\|_{r_{j}, Q_{T}}^{r_{j}} \leq C_{6}\left\{\sum_{|\alpha|=l}\left\|\mathcal{D}^{\alpha} u\right\|_{p, Q_{T}}^{p} \cdot\|u\|_{\infty, Q_{T}}^{r_{j}-p}+T\|u\|_{\infty, Q_{T}}^{r_{j}}\right\}
$$

holds with $j=1, \ldots, l-1, r_{j}=\frac{l p}{j}$. Here, $\|\cdot\|_{\infty, Q_{T}}$ is the norm of $L_{\infty}\left(Q_{T}\right)$.

The inequality (2.9) follows from the Nirenberg-Gagliardo interpolation inequality 12 for a fixed value of $t$ and a subsequent integration over $t$.

We shall study the solvability of nonlinear initial-boundary value problems with zero initial condition. To this end we need some particular subspaces of the spaces $W_{p}^{(l)}\left(Q_{T}\right)$ and $W_{p}^{(l)}\left(S_{T}\right)$ consisting of functions vanishing on $\Omega_{0}$.

For a positive and even $l$, we define $W_{p}^{(l), 0}\left(Q_{T}\right)$ to be the subspace of $W_{p}^{(l)}\left(Q_{T}\right)$ consisting of all $u \in W_{p}^{(l)}\left(Q_{T}\right)$ satisfying the zero initial conditions

$$
\frac{\partial^{k} u(x, t)}{\partial t^{k}}=0, \quad(x, t) \in \Omega_{0}, k=0,1, \ldots, \frac{l}{2}-1 .
$$

The space $W_{p}^{(l), 0}\left(S_{T}\right)$ is defined, for a non-integer $l$, to be the subspace of $W_{p}^{(l)}\left(S_{T}\right)$ consisting of all $u \in W_{p}^{(l)}\left(S_{T}\right)$ satisfying the conditions (2.10) as follows:

(i) for $k=0,1, \ldots,\left[\frac{l}{2}\right]-1$, if $p\left(\frac{l}{2}-\left[\frac{l}{2}\right]\right)<1$;

(ii) for $k=0,1, \ldots,\left[\frac{l}{2}\right]$, if $p\left(\frac{l}{2}-\left[\frac{l}{2}\right]\right)>1$.

The following properties are known (see [8, Chapter 4, Section 4]). If $\tilde{u}(x, t)$ is the extension of the function $u \in W_{p}^{(l), 0}\left(Q_{T}\right)$ on the domain $\Omega \times(-T, T)$, so that $\tilde{u}(x, t)=0,(x, t) \in \Omega \times(-T, 0)$, then the function $v(x, t)=\tilde{u}(x, t-T)$ belongs to the space $W_{p}^{(l)}\left(Q_{2 T}\right)$. An analogous property is valid for functions in $W_{p}^{(l), 0}\left(S_{T}\right)$. 
2. Formulation of the main assumptions. We denote by $\mathcal{N}$ the number $1+n+\frac{n+1}{2}$. We shall write the vectors of $\xi \in \mathcal{R}^{\mathcal{N}}$ in coordinate form as $\xi=$ $\left\{\xi_{\alpha}:|\alpha| \leq 2\right\}$. In what follows we shall always assume, without further mention, that the boundary of the domain $\Omega$ belongs to the class $C^{4}$ and that the number $p$ satisfies the condition

$$
p>\frac{n+2}{2}, \quad p \neq 3 .
$$

We shall investigate the solvability in $W_{p}^{(4)}\left(Q_{T}\right)$ of the nonlinear initial-boundary value problem (1.1)-(1.3), and assume that the following conditions are satisfied:

$\left.F_{1}\right)$ the real valued function $F(x, t, \xi)$ is defined for $(x, t) \in \overline{Q_{T}}, \xi \in \mathcal{R}^{\mathcal{N}}$, and has continuous derivatives up to the third order; moreover, $F(x, t, 0) \equiv 0$;

$F_{2}$ ) there exists a continuous nonincreasing function $\nu: \overline{\mathcal{R}_{+}} \rightarrow \mathcal{R}_{+}$such that for every $(x, t) \in \overline{Q_{T}}, \xi \in \mathcal{R}^{\mathcal{N}}, \eta \in \mathcal{R}^{n}$ the inequality

$$
\sum_{|\alpha|=2} F_{\alpha}(x, t, \xi) \eta^{\alpha} \geq \nu(|\xi|)|\eta|^{2}
$$

holds, where $\eta^{\alpha}=\eta_{1}^{\alpha_{1}} \ldots \eta_{n}^{\alpha_{n}}$ and $F_{\alpha}(x, t, \xi)=\frac{\partial}{\partial \xi_{\alpha}} F(x, t, \xi) ;$

$\left.G_{1}\right)$ the function $G(x, t, \zeta)$ is defined for $(x, t) \in \overline{S_{T}}, \zeta \in\left\{\zeta_{\alpha}:|\alpha| \leq 1\right\} \in \mathcal{R}^{n+1}$ and has continuous derivatives up to the fourth order; moreover, $G(x, t, 0) \equiv$ 0

$\left.G_{2}\right)$ for every $(x, t) \in \overline{S_{T}}, \zeta \in \mathcal{R}^{n+1}$ the inequality

$$
\sum_{|\alpha|=1} G_{\alpha}(x, t, \zeta) n_{\alpha}(x) \geq \nu(|\zeta|)
$$

holds, where $n(x)=\left\{n_{\alpha}(x):|\alpha|=1\right\}$ is the unit vector in the direction of the outward normal to $\partial \Omega$ at the point $x$ and $G_{\alpha}(x, t, \zeta)=\frac{\partial}{\partial \zeta_{\alpha}} G(x, t, \zeta)$.

The functions on the right-hand side of (1.1)-(1.3) satisfy the inclusions (1.6). We shall assume that these functions satisfy the compatibility conditions for $x \in$ $\partial \Omega, t=0$. These conditions involve the fact that the function $h$ and the derivative $\frac{\partial}{\partial t} u$, which can be determined for $t=0$ by means of the equation and initial condition, must satisfy, for $x \in \partial \Omega, t=0$, the boundary condition and the inequality which follows from the boundary condition after differentiation with respect to $t$.

We use the notation $u^{(1)}(x)=\left.\frac{\partial}{\partial t} u(x, t)\right|_{t=0}, x \in \Omega$. In view of (1.1) and (1.3), this function is determined by the equality

$$
u^{(1)}(x)=F\left(x, 0, h(x), \mathcal{D}^{1} h(x), \mathcal{D}^{2} h(x)\right)+f(x, 0) .
$$

We say that the "compatibility condition of the first order" is fulfilled for the problem (1.1)-(1.3) if for $x \in \partial \Omega$ the following equalities hold, where $G_{t}(x, t, \zeta)=$ $\frac{\partial}{\partial t} G(x, t, \zeta)$ :

$$
\begin{aligned}
& G\left(x, 0, h(x), \mathcal{D}^{1} h(x)\right)=g(x, 0) \\
& G_{t}\left(x, 0, h(x), \mathcal{D}^{1} h(x)\right)+\sum_{|\alpha| \leq 1} G_{\alpha}\left(x, 0, h(x), \mathcal{D}^{1} h(x)\right) \mathcal{D}^{\alpha} u^{(1)}(x) \\
& =\frac{\partial g(x, 0)}{\partial t}
\end{aligned}
$$

If only the first equality in (2.15) is true, we say that the "compatibility condition of zero order" is fulfilled for the problem (1.1)-(1.3). 
Definition 2.1. We say that the initial-boundary value problem (1.1)-(1.3) satisfies the "compatibility condition" if the compatibility condition of zero order is fulfilled for $\frac{n+2}{2}<p<3$, or the compatibility condition of the first order is fulfilled for $p>\frac{n+2}{2}, p>3$.

We shall reduce the problem (1.1)-(1.3) to the problem with zero initial data. In this case the fulfillment of the compatibility condition follows from the fact that the right-hand sides of the equation and the boundary condition belong to $W_{p}^{(2), 0}\left(Q_{T}\right)$ and $W_{p}^{\left(3-\frac{1}{p}\right), 0}\left(S_{T}\right)$, respectively.

We start with two elementary lemmas which will be useful in this section as well as later.

Lemma 2.4. Let $p>\frac{n+2}{2}$, and let $\Phi(x, t, v)$ be defined for $(x, t) \in Q_{T}, v=$ $\left(v_{1}, \ldots, v_{M}\right) \in \mathcal{R}^{M}$, and have continuous derivatives up to the second order. Then the operator $\widetilde{\Phi}$ defined by

$$
[\widetilde{\Phi}(v)](x, t)=\Phi(x, t, v(x, t))
$$

is bounded and continuous acting from the space $\left[W_{p}^{(2), 0}\left(Q_{T}\right)\right]^{M}$ into $W_{p}^{(2)}\left(Q_{T}\right)$, where $v(x, t)=\left(v_{1}(x, t), \ldots, v_{M}(x, t)\right)$.

Lemma 2.5. Let $p>\frac{n+2}{2}$, and let $\Psi(x, t, w)$ be defined for $(x, t) \in S_{T}, w=$ $\left(w_{1}, \ldots, w_{M}\right) \in \mathcal{R}^{M}$, and have continuous derivatives up to the third order. Then the operator $\widetilde{\Psi}$ defined by

$$
[\widetilde{\Psi}(w)](x, t)=\Psi(x, t, w(x, t))
$$

for $w(x, t)=\left(w_{1}(x, t), \ldots, w_{M}(x, t)\right)$ is bounded and continuous acting from the space $\left[W_{p}^{\left(3-\frac{1}{p}\right), 0}\left(S_{T}\right)\right]^{M}$ into $W_{p}^{\left(3-\frac{1}{p}\right), 0}\left(S_{T}\right)$.

The proofs of the two lemmas above follow from direct evaluation of the norms of the functions in the right-hand sides of (2.16) and (2.17), Lemmas 2.2 and 2.3, and the embedding theorems.

We formulate the problem with zero initial condition in the following manner: find a function $u \in W_{p}^{(4), 0}\left(Q_{T}\right)$ such that

$$
\begin{gathered}
\frac{\partial u}{\partial t}-F^{\prime}\left(x, t, u, \mathcal{D}^{1} u, \mathcal{D}^{2} u\right)=f^{\prime}(x, t), \quad(x, t) \in Q_{T}, \\
G^{\prime}\left(x, t, u, \mathcal{D}^{1} u\right)=g^{\prime}(x, t), \quad(x, t) \in S_{T},
\end{gathered}
$$

where

$$
f^{\prime} \in W_{p}^{(2), 0}\left(Q_{T}\right), g^{\prime} \in W_{p}^{\left(3-\frac{1}{p}\right), 0}\left(S_{T}\right),
$$

and the functions $F(x, t, \xi)=F^{\prime}(x, t, \xi), G(x, t, \zeta)=G^{\prime}(x, t, \zeta)$ satisfy Conditions $F_{2}$ ) and $G_{2}$ ), as well as the following smoothness conditions:

$\left.F_{3}\right)$ the function $F(x, t, \xi)$ is defined for $(x, t) \in Q_{T}, \xi=\left\{\xi_{\alpha}:|\alpha| \leq 2\right\} \in \mathcal{R}^{\mathcal{N}}$, and has continuous derivatives of the first order with respect to $\bar{\xi}_{\alpha},|\alpha| \leq 2$; moreover, $F(x, t, 0) \equiv 0$; 
$F_{4}$ ) the operators $\widetilde{F}_{\alpha}$, defined by

$$
\left[\widetilde{F}_{\alpha}(u)\right](x, t)=F_{\alpha}\left(x, t, u(x, t), \mathcal{D}^{1} u(x, t), \mathcal{D}^{2} u(x, t)\right), \quad|\alpha| \leq 2,
$$

are bounded and continuous acting from $W_{p}^{(4), 0}\left(Q_{T}\right)$ to $W_{p}^{(2)}\left(Q_{T}\right)$ with $p$ satisfying Condition (2.11) and $F_{\alpha}(x, t, \xi)$ defined in Condition $F_{2}$ );

$\left.G_{3}\right)$ the function $G(x, t, \zeta)$, defined for $(x, t) \in S_{T}, \zeta=\left\{\zeta_{\alpha}:|\alpha| \leq 1\right\} \in \mathcal{R}^{n+1}$, has continuous derivatives of the first order with respect to $\zeta_{\alpha},|\alpha| \leq 1$; moreover, $G(x, t, 0) \equiv 0$;

$\left.G_{4}\right)$ the operator $\widetilde{G}_{\beta}$, defined by

$$
\left[\widetilde{G}_{\beta}(u)\right](x, t)=G_{\beta}\left(x, t, u(x, t), \mathcal{D}^{1} u(x, t)\right),|\beta| \leq 1,(x, t) \in S_{T},
$$

is bounded and continuous acting from $W_{p}^{(4), 0}\left(Q_{T}\right)$ into $W_{p}^{\left(3-\frac{1}{p}\right), 0}\left(S_{T}\right)$ with $p$ satisfying Condition (2.11) and $G_{\beta}(x, t, \zeta)$ defined in Condition $\left.G_{2}\right)$.

Now we elaborate on the reduction of the problem (1.1)-(1.3) to the problem with zero initial condition by assuming the above conditions. Using extendability results from [5] and [8, Theorem 4.4], we define a function $v \in W_{p}^{(4), 0}\left(Q_{T}\right)$ which satisfies the initial conditions

$$
\left.\frac{\partial^{k}}{\partial t^{k}} v(x, t)\right|_{t=0}=u^{(k)}(x), \quad k=0,1
$$

and the estimate

$$
\|v\|_{p, Q_{T}}^{(4)} \leq C_{9} \sum_{k=0}^{1}\left\|u^{(k)}\right\|_{p, \Omega}^{\left(4-2 k-\frac{2}{p}\right)}
$$

where $u^{(0)}(x)=h(x)$ and $u^{(k)}(x)$ is the function defined by (2.14).

We now introduce a new function $u_{1}(x, t)$ by the equality

$$
u_{1}(x, t)=u(x, t)-v(x, t) .
$$

If $u(x, t)$ is the solution of the problem (1.1)-(1.3), then $u_{1}(x, t)$ is the solution of the boundary value problem

$$
\begin{gathered}
\frac{\partial u_{1}}{\partial t}-F_{1}\left(x, t, u_{1}, \mathcal{D}^{1} u_{1}, \mathcal{D}^{2} u_{1}\right)=f_{1}(x, t), \quad(x, t) \in Q_{T}, \\
G_{1}\left(x, t, u_{1}, \mathcal{D}^{1} u_{1}\right)=g_{1}(x, t), \quad(x, t) \in S_{T},
\end{gathered}
$$

where

$$
\begin{aligned}
& f_{1}(x, t)=f(x, t)-\frac{\partial v(x, t)}{\partial t}+F\left(x, t, v(x, t), \mathcal{D}^{1} v(x, t), \mathcal{D}^{2} v(x, t)\right), \\
& g_{1}(x, t)=g(x, t)-G\left(x, t, v(x, t), \mathcal{D}^{1} v(x, t)\right),
\end{aligned}
$$

and the functions $F_{1}, G_{1}$ are easy to evaluate.

We can check that if Conditions $\left.\left.\left.F_{1}\right), F_{2}\right), G_{1}\right), G_{2}$ ) and the compatibility conditions are satisfied, then the following three conditions hold.

a) the functions $F_{1}(x, t, \xi), G_{1}(x, t, \zeta)$, defined by (2.46), satisfy Conditions $\left.F_{2}\right), G_{2}$ ) (with some function $\nu_{1}(s)$ instead of $\nu(s)$ ) and Conditions $F_{3}$ ), $F_{4}$ ), $\left.\left.G_{3}\right), G_{4}\right)$; 
b) the functions $u_{1}(x, t), f_{1}(x, t), g_{1}(x, t)$, defined by (2.25), (2.28), satisfy the inclusions

$$
u_{1} \in W_{p}^{(4), 0}\left(Q_{T}\right), f_{1} \in W_{p}^{(2), 0}\left(Q_{T}\right), g_{1} \in W_{p}^{\left(3-\frac{1}{p}\right), 0}\left(S_{T}\right) ;
$$

c) $u(x, t)$ is a solution of the problem (1.1)-(1.3) if and only if $u_{1}(x)$ is a solution of the problem (2.26), (2.27).

Consequently, we only need to study the solvability of the boundary value problem with zero initial data.

Remark 2.1. We should note here that the problem (1.1)-(1.3) can be formulated under weaker smoothness conditions on the functions $F(x, t, \xi), G(x, t, \zeta)$ than Conditions $\left.\left.F_{1}\right), G_{1}\right)$. This can be achieved by replacing $\left.F_{1}\right), G_{1}$ ) by the conditions $\left.\left.\left.\left.F_{3}\right), F_{4}\right), G_{3}\right), G_{4}\right)$.

\section{ReduCtion to the operator EQUATION AND FORMULATION OF THE MAIN RESULTS}

We shall construct a nonlinear operator $A: W_{p}^{(4), 0}\left(Q_{T}\right) \rightarrow\left[W_{p}^{(4), 0}\left(Q_{T}\right)\right]^{*}$ satisfying Condition $(S)_{+}$(see [13, 14]) and such that the solvability of the boundary value problem $(2.18),(2.19)$ is equivalent to the solvability of the operator equation

$$
A u=0, \quad u \in W_{p}^{(4), 0}\left(Q_{T}\right) .
$$

By $\left[W_{p}^{(4), 0}\left(Q_{T}\right)\right]^{*}$ we mean the dual space of the space $W_{p}^{(4), 0}\left(Q_{T}\right)$.

We shall omit the primes in our notation for the problem (2.18), (2.19). Thus, we shall consider the problem

$$
\begin{gathered}
\frac{\partial u}{\partial t}-F\left(x, t, u, \mathcal{D}^{1} u, \mathcal{D}^{2} u\right)=f(x, t), \quad(x, t) \in Q_{T}, u \in W_{p}^{(4), 0}\left(Q_{T}\right), \\
G\left(x, t, u, \mathcal{D}^{1} u\right)=g(x, t), \quad(x, t) \in S_{T},
\end{gathered}
$$

where

$$
f \in W_{p}^{(2), 0}\left(Q_{T}\right), \quad g \in W_{p}^{\left(3-\frac{1}{p}\right), 0}\left(S_{T}\right),
$$

and the functions $F(x, t, \xi), G(x, t, \zeta)$ satisfy conditions $\left.\left.\left.F_{2}\right)-F_{4}, G_{2}\right)-G_{4}\right)$, respectively, while $p$ satisfies (2.11).

We introduce the operators $L(u), B(u)$ by

$$
\begin{gathered}
L(u) \phi=\sum_{|\alpha| \leq 2} F_{\alpha}\left(x, t, u, \mathcal{D}^{1} u, \mathcal{D}^{2} u\right) \mathcal{D}^{\alpha} \phi, \quad(x, t) \in Q_{T}, \\
B(u) \phi=\sum_{|\beta| \leq 1} G_{\beta}\left(x, t, u, \mathcal{D}^{1} u\right) \mathcal{D}^{\beta} \phi, \quad(x, t) \in S_{T},
\end{gathered}
$$

where $u, \phi \in W_{p}^{(4), 0}\left(Q_{T}\right)$ and $F_{\alpha}(x, t, \xi), G_{\beta}(x, t, \zeta)$ are defined in Conditions $\left.\left.F_{2}\right), G_{2}\right)$. It is clear that, for a fixed function $u$, the operators $L(u), B(u)$ are linear.

Lemma 3.1. Assume that Conditions (2.11), $\left.\left.\left.F_{2}\right)-F_{4}\right), G_{2}\right)-G_{4}$ ) are satisfied. Then there exists a continuous nondecreasing function $\omega_{1}: \overline{\mathcal{R}_{+}} \rightarrow \mathcal{R}_{+}$such 
that the inequalities

$$
\begin{aligned}
& \|L(u) \phi\|_{p, Q_{T}}^{(2)} \leq \omega_{1}\left(\|u\|_{p, Q_{T}}^{(4)}\right) \cdot\|\phi\|_{p, Q_{T}}^{(4)} \\
& \|B(u) \phi\|_{p, S_{T}}^{\left(3-\frac{1}{p}\right)} \leq \omega_{1}\left(\|u\|_{p, Q_{T}}^{(4)}\right) \cdot\|\phi\|_{p, Q_{T}}^{(4)}
\end{aligned}
$$

hold for arbitrary functions $u, \phi \in W_{p}^{(4), 0}\left(Q_{T}\right)$.

Proof. We set $R=\|u\|_{p, Q_{T}}^{(4)}$. By Condition $F_{4}$ ), the norms of the coefficients of the linear operator $L(u)$ in $W_{p}^{(2)}\left(Q_{T}\right)$ are bounded above by $C^{\prime}(R)$, where $C^{\prime}$ is a nondecreasing function. Using Lemma 2.4, we obtain the estimate

$$
\|L(u) \phi\|_{p, Q_{T}}^{(2)} \leq C^{\prime \prime}(R), \quad \text { for }\|\phi\|_{p, Q_{T}}^{(4)}=1
$$

where $C^{\prime \prime}$ is a nondecreasing function. From the last estimate we immediately obtain (3.7). The proof of (3.8) is similar, using Lemmas 2.1 and 2.5.

Noting that

$$
\begin{aligned}
F\left(x, t, u, \mathcal{D}^{1} u, \mathcal{D}^{2} u\right) & =\int_{0}^{1} L(s u) u d s, \\
G\left(x, t, u, \mathcal{D}^{1} u\right) & =\int_{0}^{1} B(s u) u d s,
\end{aligned}
$$

we obtain from Lemma 3.1 the following corollary.

Corollary 3.1. Assume that the conditions of Lemma 3.1 are satisfied. Then for $u \in W_{p}^{(4), 0}\left(Q_{T}\right)$ the inequalities

$$
\begin{gathered}
\left\|F\left(\cdot, \cdot, u, \mathcal{D}^{1} u, \mathcal{D}^{2} u\right)\right\|_{p, Q_{T}}^{(2)} \leq \omega_{1}\left(\|u\|_{p, Q_{T}}^{(4)}\right) \cdot\|u\|_{p, Q_{T}}^{(4)} \\
\left\|G\left(\cdot, \cdot, u, \mathcal{D}^{2} u\right)\right\|_{p, S_{T}}^{\left(3-\frac{1}{p}\right)} \leq \omega_{1}\left(\|u\|_{p, Q_{T}}^{(4)}\right) \cdot\|u\|_{p, Q_{T}}^{(4)}
\end{gathered}
$$

hold with the same function $\omega_{1}$ as in Lemma 3.1.

We now introduce the operators

$$
\begin{aligned}
& A_{E}: W_{p}^{(4), 0}\left(Q_{T}\right) \times W_{p}^{(2), 0}\left(Q_{T}\right) \rightarrow\left[W_{p}^{(4), 0}\left(Q_{T}\right)\right]^{*}, \\
& A_{B}: W_{p}^{(4), 0}\left(Q_{T}\right) \times W_{p}^{\left(3-\frac{1}{p}\right), 0}\left(S_{T}\right) \rightarrow\left[W_{p}^{(4), 0}\left(Q_{T}\right)\right]^{*},
\end{aligned}
$$

the first of which corresponds to the differential equation (3.2) and the second to the boundary condition (3.3). We define, for $u, \phi \in W_{p}^{(4), 0}\left(Q_{T}\right), v \in W_{p}^{(2), 0}\left(Q_{T}\right)$,

$$
\begin{aligned}
\left\langle A_{E}(u, v), \phi\right\rangle= & \int_{Q_{T}} \sum_{2 s+|\alpha| \leq 2} \psi_{p}\left[\left(\frac{\partial}{\partial t}\right)^{s} \mathcal{D}^{\alpha} v(x, t)\right] \\
& \cdot\left(\frac{\partial}{\partial t}\right)^{s} \mathcal{D}^{\alpha}\left[\frac{\partial \phi(x, t)}{\partial t}-L(u) \phi(x, t)\right] d x d t,
\end{aligned}
$$

where the function $\psi_{p}: \mathcal{R} \rightarrow \mathcal{R}$ is defined by

$$
\psi_{p}(s)=|s|^{p-2} \cdot s .
$$

In (3.11) and later, we use, for $\phi \in W_{p}^{(4), 0}\left(Q_{T}\right), l \in\left[W_{p}^{(4), 0}\left(Q_{T}\right)\right]^{*}$, the symbol $\langle l, \phi\rangle$ to denote the value of the functional $l$ at the function $\phi$. From Lemma 3.2, 
which will be formulated below, it follows that the operator $A_{E}$ is well defined by (3.11).

For the definition of the operator $A_{B}$ we introduce local coordinate systems near the boundary $\partial \Omega$, which belongs, by our assumption, to the class $\mathcal{C}^{4}$. Let $n(\xi)$ be the unit vector in the direction of the outward normal to $\partial \Omega$ at the point $\xi \in \partial \Omega$. A cartesian coordinate system $\{y\}$, with the origin at $\xi$ and its $y_{n}$-axis directed along $n(\xi)$, is called a "local coordinate system". We choose a finite collection $\left\{\mathcal{U}_{i}\right\}, i=$ $1, \ldots, I$, of open sets such that (a) $\bigcup_{i=1}^{I} \mathcal{U}_{i} \supset \partial \Omega$, and (b) for some point $\xi_{i} \in \mathcal{U}_{i} \cap \partial \Omega$ and some positive number $d$ the set $\mathcal{U}_{i} \cap \partial \Omega$ is given in a local coordinate system $\{y\}$ at the point $\xi_{i}$ by the equation

$$
y_{n}=h_{i}\left(y^{\prime}\right), \quad y^{\prime}=\left(y_{1}, \ldots, y_{n-1}\right) \in B^{\prime}(d)=\left\{y^{\prime}:\left|y^{\prime}\right|<d\right\},
$$

where $h_{i} \in \mathcal{C}^{4}\left(B^{\prime}(d)\right)$. For the function $u(x, t)$ defined on $S_{T}$ we define $u^{(i)}\left(y^{\prime}, t\right)$, $\left(y^{\prime}, t\right) \in D_{T}(d) \equiv B^{\prime}(d) \times(0, T)$, by $u^{(i)}\left(y^{\prime}, t\right)=u(x, t)$, for $x=\phi\left(y^{\prime}, h_{i}\left(y^{\prime}\right)\right)$, where $\phi$ is the transformation corresponding to the system $\{y\}$ at $\xi_{i}$.

We define, for $u, \phi \in W_{p}^{(4), 0}\left(Q_{T}\right)$ and $w \in W_{p}^{\left(3-\frac{1}{p}\right), 0}\left(S_{T}\right)$,

$$
\left\langle A_{B}(u, w), \phi\right\rangle=\sum_{k=1}^{3}\left\langle A_{B}^{(k)}(u, w), \phi\right\rangle,
$$

where

$$
\begin{aligned}
& \left\langle A_{B}^{(1)}(u, w), \phi\right\rangle=\sum_{i=1}^{I} \sum_{2 s+|\beta| \leq 2}^{\prime} \int_{0}^{T} \int_{B^{\prime}(d)} \psi_{p}\left[\left(\frac{\partial}{\partial t}\right)^{s} \mathcal{D}_{y}^{\beta} w^{(i)}\left(y^{\prime}, t\right)\right] \\
& \cdot\left(\frac{\partial}{\partial t}\right)^{s} \mathcal{D}_{y}^{\beta}[B(u) \phi]^{(i)}\left(y^{\prime}, t\right) d t d y^{\prime}, \\
& \left\langle A_{B}^{(2)}(u, w), \phi\right\rangle=\sum_{i=1}^{I} \sum_{2 s+|\beta|=2}^{\prime} \int_{0}^{T} d t \\
& \cdot \int_{B^{\prime}(d)} d y^{\prime} \int_{B^{\prime}(d)} \psi_{p}\left[\left(\frac{\partial}{\partial t}\right)^{s} \mathcal{D}_{y}^{\beta} w^{(i)}\left(y^{\prime}, t\right)-\left(\frac{\partial}{\partial t}\right)^{s} \mathcal{D}_{z}^{\beta} w^{(i)}\left(z^{\prime}, t\right)\right] \\
& \cdot\left\{\left(\frac{\partial}{\partial t}\right)^{s} \mathcal{D}_{y}^{\beta}[B(u) \phi]^{(i)}\left(y^{\prime}, t\right)-\left(\frac{\partial}{\partial t}\right)^{s} \mathcal{D}_{z}^{\beta}[B(u) \phi]^{(i)}\left(z^{\prime}, t\right)\right\} \\
& \cdot \frac{d z^{\prime}}{\left|y^{\prime}-z^{\prime}\right|^{n+p-2}}, \\
& \left\langle A_{B}^{(3)}(u, w), \phi\right\rangle=\sum_{i=1}^{I} \sum_{j=1}^{2} \sum_{2 s+|\beta|=j}^{\prime} \int_{B^{\prime}(d)} d y^{\prime} \int_{0}^{T} d t \int_{0}^{T} \\
& \quad \psi_{p}\left[\left(\frac{\partial}{\partial t}\right)^{s} \mathcal{D}_{y}^{\beta} w^{(i)}\left(y^{\prime}, t\right)-\left(\frac{\partial}{\partial \tau}\right)^{s} \mathcal{D}_{y^{\prime}}^{\beta} w^{(i)}\left(y^{\prime}, \tau\right)\right] \\
& \quad \cdot\left\{\left(\frac{\partial}{\partial t}\right)^{s} \mathcal{D}_{y}^{\beta}[B(u) \phi]^{(i)}\left(y^{\prime}, t\right)-\left(\frac{\partial}{\partial \tau}\right)^{s} \mathcal{D}_{y}^{\beta}[B(u) \phi]^{(i)}\left(y^{\prime}, \tau\right)\right\} \\
& \quad \cdot \frac{d \tau}{|t-\tau|^{p(j)}},
\end{aligned}
$$


where $p(1)=p+\frac{1}{2}, p(2)=\frac{1}{2}(p+1)$ and $\sum^{\prime}$ denotes the sum over multi-indices $\beta$ with last component $\beta_{n}$ equal to zero. The function $\psi_{p}$ in (3.14)-(3.16) is defined in (3.12).

From the following lemma we obtain that the right-hand sides of (3.11) and (3.13) generate continuous linear functionals with respect to $\phi \in W_{p}^{(4), 0}\left(Q_{T}\right)$ for fixed functions $u, v, w$ that make the operators $A_{E}, A_{B}$ well defined.

Lemma 3.2. Assume that the conditions of Lemma 3.1 are satisfied. Then there exists a continuous nondecreasing function $\omega_{2}: \overline{\mathcal{R}_{+}} \rightarrow \mathcal{R}_{+}$such that the inequalities

$$
\begin{gathered}
\left|\left\langle A_{E}(u, v), \phi\right\rangle\right| \leq \omega_{2}\left(\|u\|_{p, Q_{T}}^{(4)}\right) \cdot\left[\|v\|_{p, Q_{T}}^{(2)}\right]^{p-1} \cdot\|\phi\|_{p, Q_{T}}^{(4)}, \\
\left|\left\langle A_{B}(u, w), \phi\right\rangle\right| \leq \omega_{2}\left(\|u\|_{p, Q_{T}}^{(4)}\right) \cdot\left[\|w\|_{p, S_{T}}^{\left(3-\frac{1}{p}\right)}\right]^{p-1} \cdot\|\phi\|_{p, Q_{T}}^{(4)}
\end{gathered}
$$

hold.

Proof. The estimates (3.17) follow immediately from Lemma 3.1 and Hölder's inequality.

We now define the operator $A: W_{p}^{(4), 0}\left(Q_{T}\right) \rightarrow\left[W_{p}^{(4), 0}\left(Q_{T}\right)\right]^{*}$ by

$$
\begin{aligned}
A u= & A_{E}\left(u, \frac{\partial u}{\partial t}-F\left(x, t, u, \mathcal{D}^{1} u, \mathcal{D}^{2} u\right)-f(x, t)\right) \\
& +A_{B}\left(u, G\left(x, t, u, \mathcal{D}^{1} u\right)-g(x, t)\right) .
\end{aligned}
$$

Taking into consideration Corollary 3.1 and Lemma 3.2, we obtain that the operator $A$ is well defined.

For the formulation of the main properties of the operator $A$ we need the notion of Condition $(S)_{+}$, which we state below for an arbitrary reflexive Banach space $X$ with dual space $X^{*}$. For arbitrary elements $u \in X$ and $l \in X^{*}$ we denote by $\langle l, u\rangle$ the value of the functional $l$ at $u$.

Definition 3.1. We say that an operator $H: X \rightarrow X^{*}$ satisfies Condition $(S)_{+}$ on $X$ if for every sequence $\left\{u_{j}\right\} \subset X$ satisfying the conditions

$$
u_{j} \rightarrow u_{0}, \quad \limsup _{j \rightarrow \infty}\left\langle H u_{j}, u_{j}-u_{0}\right\rangle \leq 0,
$$

for some element $u_{0} \in X$, we have $u_{j} \rightarrow u_{0}$.

The fundamental results of this paper are given in the following theorems.

Theorem 3.1. Assume that Conditions (2.11), (3.4), $\left.\left.\left.F_{2}\right)-F_{4}\right), G_{2}\right)-G_{4}$ ) are satisfied. Then the operator A, defined by (3.18), is bounded and continuous, and satisfies Condition $(S)_{+}$on $W_{p}^{(4), 0}\left(Q_{T}\right)$.

Theorem 3.2. Assume that the conditions of Theorem 3.1 are satisfied. Then a function $u \in W_{p}^{(4), 0}\left(Q_{T}\right)$ is a solution of the boundary value problem (3.2), (3.3) if and only if it is a solution of the operator equation (3.1) with the operator $A$ defined by (3.18).

Theorems 3.1 and 3.2 will be proved in Section 5 after we establish, in Section 4 , a priori estimates and solvability results for linear parabolic problems in Sobolev spaces. In addition, by using the results of Section 4, we shall prove the following uniqueness theorem. 
Theorem 3.3. Assume that the conditions of Theorem 3.1 are satisfied. Then the boundary value problem (3.2), (3.3) can have at most one solution.

Obviously, a uniqueness theorem analogous to Theorem 3.3 is also valid for the initial-boundary value problem (1.1)-(1.3) if Conditions $\left.\left.(2.11),(1.6), F_{1}\right), F_{2}\right)$, $\left.G_{1}\right), G_{2}$ ) and the compatibility condition are satisfied. This follows immediately from the reduction of the problem (1.1)-(1.3) to the problem with zero initial condition in Section 2 above.

On the basis of Theorem 3.1 we can now see the feasibility of applying the degree theory of the monograph [14] to the operator $A$. Let $\mathcal{D}$ be a bounded open set in $W_{p}^{(4), 0}\left(Q_{T}\right)$ and let

$$
A u \neq 0, \quad u \in \partial \Omega,
$$

where the operator $A$ is defined by (3.18). Then from [14] we see that the degree $\operatorname{Deg}(A, \overline{\mathcal{D}}, 0)$ is well defined with respect to $\overline{\mathcal{D}}$ and the point $0 \in\left[W_{p}^{(4), 0}\left(Q_{T}\right)\right]^{*}$. This degree function is integer-valued.

Theorem 3.4. Assume that the conditions of Theorem 3.1 are satisfied. Then for every open and bounded set $\mathcal{D} \subset W_{p}^{(4), 0}\left(Q_{T}\right)$, such that Condition (3.20) is satisfied, the degree $\operatorname{Deg}(A, \overline{\mathcal{D}}, 0)$ of the operator $A$ in (3.18) is well defined.

The degree of the operator $A$ provides us with a topological characteristic for the nonlinear parabolic problem (3.2), (3.3). Using the degree theory from [13] and [14], it is possible to study the solvability of this problem via topological methods.

We formulate below two results which will be proved by topological methods. We first state a result on the local solvability of the problem (1.1)-(1.3).

Theorem 3.5. Assume that Conditions (2.11), $\left.\left.\left.F_{1}\right), F_{2}\right), G_{1}\right), G_{2}$ ) and the compatibility condition are satisfied, and let $\mathcal{K}$ be a positive number. Then there exists a positive number $T_{0}$ such that the initial-boundary value problem (1.1)-(1.3) has a unique solution $u \in W_{p}^{(4), 0}\left(Q_{T}\right)$ if the inequalities

$$
\|f\|_{p, Q_{T}}^{(2)} \leq \mathcal{K},\|g\|_{p, S_{T}}^{\left(3-\frac{1}{p}\right)} \leq \mathcal{K},\|h\|_{p, Q_{T}}^{\left(4-\frac{2}{p}\right)} \leq \mathcal{K}
$$

are satisfied and $0<T<T_{0}$.

The following theorem contains a result on the global solvability of the nonlinear problem provided that we have an a priori estimate for a solution of it. We include the problem (3.2), (3.3) in the parametric family of similar problems with functions $F_{\tau}(x, t, \xi), G_{\tau}(x, t, \zeta), \tau \in[0,1]$, such that

$$
F(x, t, \xi)=F_{1}(x, t, \xi), G(x, t, \zeta)=G_{1}(x, t, \zeta) .
$$

In particular, this family can be chosen as follows:

$$
\begin{aligned}
& F_{\tau}(x, t, \xi)=\tau F(x, t, \xi)+(1-\tau) \sum_{|\alpha| \leq 2} F_{\alpha}(x, t, 0) \xi_{\alpha}, \\
& G_{\tau}(x, t, \zeta)=\tau G(x, t, \zeta)+(1-\tau) \sum_{|\beta| \leq 1} G_{\beta}(x, t, 0) \zeta_{\beta} .
\end{aligned}
$$

Theorem 3.6. Let $F(\tau, x, t, \xi), G(\tau, y, t, \zeta)$ be defined and continuous for $\tau \in$ $[0,1], x \in \bar{\Omega}, y \in \partial \Omega, t \in[0, T], \xi \in \mathcal{R}^{\mathcal{N}}, \zeta \in \mathcal{R}^{n+1}$. Assume that for every 
$\tau \in[0,1]$ the functions

$$
F_{\tau}(x, t, \xi)=F(\tau, x, t, \xi), G_{\tau}(y, t, \zeta)=G(\tau, y, t, \zeta)
$$

satisfy Conditions $\left.\left.\left.F_{2}\right)-F_{4}\right), G_{2}\right)-G_{4}$ ) of Section 2, respectively, and assume that (2.11) and (3.4) hold. In addition, assume that for the given functions $f, g$ there exists a constant $R$ such that for an arbitrary possible solution $u_{\tau} \in W_{p}^{(4), 0}\left(Q_{T}\right)$ of the problem

$$
\begin{gathered}
\frac{\partial u}{\partial t}-F_{\tau}\left(x, t, u, \mathcal{D}^{1} u, \mathcal{D}^{2} u\right)=\tau f(x, t), \quad(x, t) \in Q_{T}, \\
G_{\tau}\left(x, t, u, \mathcal{D}^{1} u\right)=\tau g(x, t), \quad(x, t) \in S_{T},
\end{gathered}
$$

the a priori estimate

$$
\left\|u_{\tau}\right\|_{p, Q_{T}}^{(4)} \leq R
$$

is valid. Then the problem (3.2), (3.3) has a unique solution $u \in W_{p}^{(4), 0}\left(Q_{T}\right)$.

Theorems 3.3, 3.5 and 3.6 will be proved in Section 6. As a particular case of Theorem 3.6 we obtain the solvability of coercive nonlinear parabolic problems. We say that the initial-boundary value problem (3.2), (3.3) satisfies a coercivity condition if

$$
\begin{aligned}
& \lim _{\substack{\|u\|_{p, Q_{T}}^{(4)} \rightarrow \infty \\
=+\infty}}\left\{\left\|\frac{\partial u}{\partial t}-F\left(\cdot, \cdot, u, \mathcal{D}^{1} u, \mathcal{D}^{2} u\right)\right\|_{p, Q_{T}}^{(2)}+\left\|G\left(\cdot, \cdot, u, \mathcal{D}^{1} u\right)\right\|_{p, S_{T}}^{\left(3-\frac{1}{p}\right)}\right\} \\
& =+\infty
\end{aligned}
$$

From Theorem 3.6, with $F_{\tau}(x, t, \xi)=F(x, t, \xi), G_{\tau}(x, t, \zeta)=G(x, t, \zeta)$, we have the following corollary.

Corollary 3.2. Assume that Conditions (2.11), $\left.\left.\left.F_{2}\right)-F_{4}\right), G_{2}\right)-G_{4}$ ) are satisfied and the problem (3.2), (3.3) satisfies the coercivity condition (3.29). Then the problem (3.2), (3.3) has a solution $u \in W_{p}^{(4), 0}\left(Q_{T}\right)$ for arbitrary functions $f \in W_{p}^{(2), 0}\left(Q_{T}\right), g \in W_{p}^{\left(3-\frac{1}{p}\right), 0}\left(S_{T}\right)$.

\section{A PRIORI ESTIMATES FOR LINEAR PARABOLIC PROBLEMS WITH COEFFICIENTS FROM SOBOLEV SPACES}

The study of the properties of the operator $A$, introduced in the preceding section, is based on results involving the solvability of and estimates for linear parabolic problems with coefficients from Sobolev spaces which are proved in this section. Let us consider the initial-boundary value problem

$$
\begin{gathered}
L u \equiv \frac{\partial u}{\partial t}-\sum_{|\alpha| \leq 2} a_{\alpha}(x, t) \mathcal{D}^{\alpha} u=f(x, t), \quad(x, t) \in Q_{T}, \\
B u \equiv \sum_{|\beta| \leq 1} b_{\beta}(x, t) \mathcal{D}^{\beta} u=g(x, t), \quad(x, t) \in S_{T}, \\
u(x, 0)=h(x), \quad x \in \Omega .
\end{gathered}
$$

We assume that the domain $\Omega$ is a bounded set in $\mathcal{R}^{n}$ and that its boundary belongs to the class $\mathcal{C}^{4}$. We also assume that the following conditions are satisfied:

$\left.a_{1}\right)$ The functions $a_{\alpha}(x, t),|\alpha| \leq 2$, are defined for $(x, t) \in Q_{T}$ and belong to the space $W_{p}^{(2)}\left(Q_{\bar{T}}\right)$; 
$\left.a_{2}\right)$ there exists a positive constant $\nu_{0}$ such that for every $(x, t) \in Q_{\bar{T}}, \eta \in \mathcal{R}^{n}$ we have

$$
\sum_{|\alpha|=2} a_{\alpha}(x, t) \eta^{\alpha} \geq \nu_{0}|\eta|^{2}
$$

$\left.b_{1}\right)$ the functions $b_{\beta}(x, t),|\beta| \leq 2$, are defined for $(x, t) \in S_{\bar{T}}$ and belong to the space $W_{p}^{\left(3-\frac{1}{p}\right)}\left(S_{\bar{T}}\right)$

$\left.b_{2}\right)$ for every $(x, t) \in S_{\bar{T}}$ the inequality

$$
\sum_{|\beta|=1} b_{\beta}(x, t) n_{\beta}(x) \geq \nu_{0}
$$

holds, where $n(x)=\left\{n_{\beta}(x):|\beta|=1\right\}$ is the unit vector in the direction of the outward normal to $\partial \Omega$ at the point $x$, and $\nu_{0}$ is the same number as in (4.4).

We also assume that

$$
f \in W_{p}^{(2)}\left(Q_{T}\right), g \in W_{p}^{\left(3-\frac{1}{p}\right)}\left(S_{T}\right), h \in W_{p}^{4-\frac{2}{p}}(\Omega)
$$

and that the compatibility condition holds for the problem (4.1)-(4.3) in the sense of Definition 2.1 for

$$
p>\frac{n+2}{2}, p \neq 3 .
$$

Theorem 4.1. Assume that the boundary of the bounded domain $\Omega$ belongs to the class $\mathcal{C}^{4}, T \in(0, \bar{T}]$, and Conditions (4.7), $\left.\left.\left.\left.a_{1}\right), a_{2}\right), b_{1}\right), b_{2}\right)$ are satisfied. Then, for any functions $f, g, h$, satisfying (4.6) and the compatibility conditions, the initialboundary value problem (4.1)-(4.3) has a unique solution $u \in W_{p}^{(4)}\left(Q_{T}\right)$. This solution satisfies the following a priori estimate:

$$
\begin{aligned}
\mathcal{K}_{1}\left\{\|f\|_{p, Q_{T}}^{(2)}+\|g\|_{p, S_{T}}^{\left(3-\frac{1}{p}\right)}\right. & \left.+\|h\|_{p, \Omega}^{\left(4-\frac{2}{p}\right)}\right\} \leq\|u\|_{p, Q_{T}}^{(4)} \\
& \leq \mathcal{K}_{2}\left\{\|f\|_{p, Q_{T}}^{(2)}+\|g\|_{p, S_{T}}^{\left(3-\frac{1}{p}\right)}+\|h\|_{p, \Omega}^{\left(4-\frac{2}{p}\right)}\right\},
\end{aligned}
$$

with positive constants $\mathcal{K}_{1}, \mathcal{K}_{2}$ depending only on $\Omega, \bar{T}, n, p, \nu_{0}$ and the norms of the coefficients $a_{\alpha}, b_{\beta}$ in the spaces $W_{p}^{(2)}\left(Q_{T}\right), W_{p}^{\left(3-\frac{1}{p}\right)}\left(S_{T}\right)$, respectively.

Remark 4.1. We use the symbols $\mathcal{K}_{i}, i=3,4, \ldots$, to denote constants that depend only on the same parameters as those of $\mathcal{K}_{1}, \mathcal{K}_{2}$ above.

Proof of Theorem 4.1. Thanks to the reduction of the problem (4.1)-(4.3) to the problem with zero initial data, shown in Section 2, it is sufficient to prove Theorem 4.1 for

$$
f \in W_{p}^{(2), 0}\left(Q_{T}\right), g \in W_{p}^{\left(3-\frac{1}{p}\right), 0}\left(S_{T}\right), h(x) \equiv 0 .
$$

We are going to show below that the solution of the problem (4.1)-(4.3) lies in the space $W_{p}^{(4), 0}\left(Q_{T}\right)$.

First, Theorem 4.1 will be proved for sufficiently small $T_{0}$. To this end, we construct a bounded operator

$$
R: W_{p}^{\left(2,3-\frac{1}{p}\right), 0}\left(Q_{T}, S_{T}\right) \equiv W_{p}^{(2), 0}\left(Q_{T}\right) \times W_{p}^{\left(3-\frac{1}{p}\right), 0}\left(S_{T}\right) \rightarrow W_{p}^{(4), 0}\left(Q_{T}\right)
$$


so that for every pair of functions $\phi=(f, g)$ satisfying condition (4.9) and every function $v \in W_{p}^{(4), 0}\left(Q_{T}\right)$ the equalities

$$
P R \phi=\phi+M \phi, R P v=v+N v
$$

hold, where

$$
P v=(L v, B v)
$$

and $M, N$ are bounded operators in the spaces $W_{p}^{\left(2,3-\frac{1}{p}\right), 0}\left(Q_{T}, S_{T}\right)$ and $W_{p}^{(4), 0}\left(Q_{T}\right)$, respectively. The norms of these two operators are bounded above by $\frac{1}{2}$ if $T_{0}$ is sufficiently small.

Let $\lambda$ be a positive number. We choose a finite cover of $\bar{\Omega}$ by open balls $B\left(x_{k}, r_{k}\right)$, $k=1, \ldots, \mathcal{K}$, so that $x_{k} \in \bar{\Omega}, r_{k} \leq \lambda$. We choose three collections of functions $\left\{\xi_{k}\right\}$, $\left\{\eta_{k}\right\},\left\{\zeta_{k}\right\}, k=1, \ldots, \mathcal{K}$, so that the following conditions are satisfied:

1) $\xi_{k}, \eta_{k}, \zeta_{k} \in \mathcal{C}_{0}^{\infty}\left(\mathcal{R}^{n}\right), k=1, \ldots, \mathcal{K}$; the supports of these functions are subsets of $B\left(x_{k}, r_{k}\right)$, respectively;

2) the identities

$$
\begin{gathered}
\sum_{k} \xi_{k}(x) \equiv 1, \quad x \in \bar{\Omega}, \\
\xi_{k}(x) \eta_{k}(x) \equiv \xi_{k}(x), \eta_{k}(x) \zeta_{k}(x) \equiv \eta_{k}(x), x \in \mathcal{R}^{n},
\end{gathered}
$$

hold for $k=1, \ldots, \mathcal{K}$; here and below the sum over $k$ is meant to be from 1 to $\mathcal{K}$;

3) for every $x \in \mathcal{R}^{n}$ and $k=1, \ldots, \mathcal{K}$, we have

$$
\begin{aligned}
& 0 \leq \xi_{k}(x) \leq 1,0 \leq \eta_{k}(x) \leq 1,0 \leq \zeta_{k}(x) \leq 1, \\
& \left|\mathcal{D}^{\alpha} \xi_{k}(x)\right|+\left|\mathcal{D}^{\alpha} \eta_{k}(x)\right|+\left|\mathcal{D}^{\alpha} \zeta_{k}(x)\right| \leq C_{0} \lambda^{-|\alpha|}, \quad \text { for }|\alpha| \leq 4,
\end{aligned}
$$

with the constant $C_{0}$ depending only on $n$.

We may also make the above choice so that the estimate

$$
\sum_{k} \chi_{k}(x) \leq C_{1}
$$

holds, where the constant $C_{1}$ is independent of $\lambda$ and $\chi_{k}$ is the characteristic function of the ball $B\left(x_{k}, \lambda\right)$. We assume that $\lambda$ is sufficiently small to guarantee that $B\left(x_{k}, r_{k}\right) \cap \partial \Omega \subset U_{i}$, for some $i=i(k), 1 \leq i \leq I$, where $\left\{U_{i}\right\}$ is the covering of $\partial \Omega$ which was chosen for the definition of the operator $A_{B}$ by (3.13)-(3.16).

We introduce the operator $R^{(k)}$ which maps the pair $(f, g)$ satisfying $(4.6)$ to the solution of the initial-boundary value problem

$$
\begin{gathered}
L_{0}^{(k)} v \equiv \frac{\partial u}{\partial t}-\sum_{|\alpha|=2} a_{\alpha}\left(x_{k}, 0\right) \mathcal{D}^{\alpha} v=f(x, t), \quad(x, t) \in Q_{T}, \\
B_{0}^{(k)} v \equiv \sum_{|\beta|=1} b_{\beta}\left(x_{k}, 0\right) \mathcal{D}^{\beta} v=g(x, t), \quad(x, t) \in S_{T}, \\
v(x, 0)=0, \quad x \in \Omega .
\end{gathered}
$$

The unique solution $v=R^{(k)}(f, g)$ of this problem exists in $W_{p}^{(4), 0}\left(Q_{T}\right)$, and the a priori estimates 


$$
\|v\|_{p, Q_{T}}^{(4)} \leq \mathcal{K}_{3}\left\{\|f\|_{p, Q_{T}}^{(2)}+\|g\|_{p, S_{T}}^{\left(3-\frac{1}{p}\right)}\right\}
$$

are valid (see, e.g., [8], [15]).

We define the operator $R$, which acts according to (4.10), by

$$
\begin{aligned}
R \phi & =\sum_{k} \xi_{k} v_{k}, \\
v_{k} & =R^{(k)}\left(\eta_{k} f, \eta_{k} g\right),
\end{aligned}
$$

where $\phi=(f, g)$. We are going to verify that under this definition of $R$ the equalities (4.11) hold true and the norms of the operators $M, N$ are sufficiently small if $T_{0}$ is small enough. We use the notation

$$
M_{1} \phi=L R \phi-f, \quad M_{2} \phi=B R \phi-g .
$$

Using (4.13) and the definition of $v_{k}(x, t)$, we have

$$
\begin{aligned}
& M_{1} \phi=\sum_{k}\left\{L\left(\xi_{k} v_{k}\right)-\xi_{k} L v_{k}+\xi_{k}\left(L v_{k}-L_{0}^{(k)} v_{k}\right)\right\}, \\
& M_{2} \phi=\sum_{k}\left\{B\left(\xi_{k} v_{k}\right)-\xi_{k} B v_{k}+\xi_{k}\left(B v_{k}-B_{0}^{(k)} v_{k}\right\} .\right.
\end{aligned}
$$

We interrupt this proof for two lemmas.

Lemma 4.1. Assume that the conditions of Theorem 4.1 are satisfied. Then there exist positive numbers $\lambda_{1}, \kappa_{1}$, depending only on the same parameters as those of $\mathcal{K}_{1}, \mathcal{K}_{2}$ of Theorem 4.1, such that for

$$
T=\lambda^{2} \kappa, \quad 0<\lambda \leq \lambda_{1}, \quad 0<\kappa \leq \min \left\{1, \kappa_{1}\right\},
$$

the inequality

$$
\left(\left\|M_{1} \phi\right\|_{p, Q_{T}}^{(2)}\right)^{p}+\left(\left\|M_{2} \phi\right\|_{p, S_{T}}^{\left(3-\frac{1}{p}\right)}\right)^{p} \leq \frac{1}{2}\left\{\left(\|f\|_{p, Q_{T}}^{(2)}\right)^{p}+\left(\|g\|_{p, S_{T}}^{\left(3-\frac{1}{p}\right)}\right)^{p}\right\}
$$

holds.

Proof. We consider only the evaluation of the principal summands in the representation of $M_{1} \phi$ :

$$
\begin{aligned}
& M_{1}^{\prime} \phi=\sum_{k} \sum_{|\alpha|=2} \xi_{k}(x)\left[a_{\alpha}(x, t)-a_{\alpha}\left(x_{k}, 0\right)\right] \mathcal{D}^{\alpha} v_{k}(x, t), \\
& M_{1}^{\prime \prime} \phi=\sum_{k} \sum_{\substack{\alpha^{\prime}+\alpha^{\prime \prime}=\alpha \\
|\alpha|=2, \alpha^{\prime}>0}} \mathcal{D}^{\alpha^{\prime}} \xi_{k}(x) a_{\alpha}(x, t) \mathcal{D}^{\alpha^{\prime \prime}} v_{k}(x, t),
\end{aligned}
$$

and the analogous summands in the representation of $M_{2} \phi$. We assume that $T=$ $\lambda^{2} \kappa, 0<\kappa \leq 1$. Using the inequality (4.15), we obtain

$$
\left\{\left\|M_{1}^{\prime} \phi\right\|_{p, Q_{T}}^{(2)}\right\}^{p} \leq \mathcal{K}_{4} \sum_{k}\left\{I_{k}^{(1)}+I_{k}^{(2)}+\sum_{\left|\beta^{\prime}+\beta^{\prime \prime}+\beta^{\prime \prime \prime}\right| \leq 2} I_{k}^{(3)}\left(\beta^{\prime}, \beta^{\prime \prime}, \beta^{\prime \prime \prime}\right)\right\},
$$


where

$$
\begin{aligned}
& I_{k}^{(1)}=\sum_{|\alpha|=2} \int_{Q_{T}^{(k)}}\left[\xi_{k}(x) \cdot\left|\frac{\partial a_{\alpha}(x, t)}{\partial t}\right| \cdot\left|\mathcal{D}^{\alpha} v_{k}(x, t)\right|\right]^{p} d x d t, \\
& I_{k}^{(2)}=\sum_{|\alpha|=2} \int_{Q_{T}^{(k)}}\left[\xi_{k}(x)\left|a_{\alpha}(x, t)-a_{\alpha}\left(x_{k}, 0\right)\right| \cdot\left|\frac{\partial}{\partial t} \mathcal{D}^{\alpha} v_{k}(x, t)\right|\right]^{p} d x d t, \\
& I_{k}^{(3)}\left(\beta^{\prime}, \beta^{\prime \prime}, \beta^{\prime \prime \prime}\right)=\sum_{|\alpha|=2} \int_{Q_{T}^{(k)}}\left[\left|\mathcal{D}^{\beta^{\prime}} \xi_{k}(x)\right| \cdot\left|\mathcal{D}^{\beta^{\prime \prime}}\left[a_{\alpha}(x, t)-a_{\alpha}\left(x_{k}, 0\right)\right]\right|\right. \\
& \left.\cdot\left|\mathcal{D}^{\alpha+\beta^{\prime \prime \prime}} v_{k}(x, t)\right|\right]^{p} d x d t,
\end{aligned}
$$

where $Q_{T}^{(k)}=\left\{B\left(x_{k}, \lambda\right) \times(0, T)\right\} \cap Q_{T}$.

For the evaluation of the integrals in (4.29) we shall use the following estimates:

$$
\left\|\left(\frac{\partial}{\partial t}\right)^{s} \mathcal{D}^{\gamma} u\right\|_{p, Q_{T}^{(k)}} \leq \mathcal{K}_{5} T^{2-\frac{j}{2}}|u|_{P, Q_{T}^{(k)}}^{(4)}
$$

for $2 s+|\gamma|=j<4$,

$$
\left|\left(\frac{\partial}{\partial t}\right)^{s} \mathcal{D}^{\gamma} u\right|_{Q_{T}^{(k)}}^{(\delta)} \leq \mathcal{K}_{6} T^{\frac{1}{2}\left(4-j-\frac{n+2}{p}-\delta\right)} \cdot|u|_{p, Q_{T}^{(k)}}^{(\delta)},
$$

and

$$
\left|\left(\frac{\partial}{\partial t}\right)^{s} \mathcal{D}^{\gamma} u\right|_{Q_{T}^{(k)}}^{(0)} \leq \mathcal{K}_{7} T^{\frac{\delta}{2}} \cdot\left|\left(\frac{\partial}{\partial t}\right)^{s} \mathcal{D}^{\gamma} u\right|_{Q_{T}^{(k)}}^{(\delta)},
$$

for $2 s+|\gamma|=j, 0<\delta<4-j-\frac{n+2}{p}$ and any function $u \in W_{p}^{(4), 0}\left(Q_{T}^{(k)}\right)$.

The inequalities (4.30), (4.31) follow from Lemma 2.2 and Poincaré's inequality, while (4.32) follows from the definition of the space $W_{p}^{(4), 0}\left(Q_{T}^{(k)}\right)$.

Using Lemma 2.3 and (4.30), (4.31), we obtain the estimate

$$
\left\|\left(\frac{\partial}{\partial t}\right)^{s} \mathcal{D}^{\gamma} u\right\|_{2 p, Q_{T}^{(k)}}^{2 p} \leq \mathcal{K}_{8} T^{\frac{p}{2}\left(2-\frac{n+2}{p}\right)}\left\{|u|_{p, Q_{T}^{(k)}}^{(4)}\right\}^{2 p},
$$

for $2 s+|\gamma|=3, u \in W_{p}^{(4), 0}\left(Q_{T}^{(k)}\right)$.

Now we are able to find upper bounds for the summands on the right-hand side of (4.28). Using (4.30)-(4.32) for the function $v_{k}(x, t)$, we obtain the estimate

$$
I_{k}^{(1)}+\sum_{\left|\beta^{\prime \prime}\right| \leq 2} I_{k}^{(3)}\left(0, \beta^{\prime \prime}, 0\right) \leq K_{9} T^{\frac{p}{2}\left(2-\frac{n+2}{p}\right)} \cdot\left\{\left|v_{k}\right|_{p, Q_{T}^{(k)}}^{(4)}\right\}^{p} .
$$

From Condition $\left.a_{1}\right),(4.7)$ and Lemma 2.2 it follows that $a_{\alpha} \in C^{\left(\delta_{0}\right)}\left(\overline{Q_{T_{0}}}\right)$ with $0<\delta_{0}<2-\frac{n+2}{p}$. Using these and (4.14), (4.30), we have the estimate

$$
I_{k}^{(2)}+\sum_{\left|\beta^{\prime}+\beta^{\prime \prime \prime}\right| \leq 2} I_{k}^{(3)}\left(\beta^{\prime}, 0, \beta^{\prime \prime \prime}\right) \leq K_{10} \lambda^{\rho \delta_{0}}\left\{\left|v_{k}\right|_{p, Q_{T}^{(k)}}^{(4)}\right\}^{p} .
$$


The inequality

$$
\sum_{\left|\beta^{\prime \prime}\right|=1} \sum_{\left|\beta^{\prime}+\beta^{\prime \prime \prime}\right|=1} I_{k}^{(3)}\left(\beta^{\prime}, \beta^{\prime \prime}, \beta^{\prime \prime \prime}\right) \leq \mathcal{K}_{11} T^{\frac{p}{4}\left(2-\frac{n+2}{p}\right)}\left\{\left|v_{k}\right|_{p, Q_{T}^{(k)}}^{(4)}\right\}
$$

can be obtained from Lemma 2.3 (for $\mathcal{D}^{\beta^{\prime \prime}} a_{\alpha}(x, t)$ ), the estimates (4.30), (4.33) for the function $v_{k}(x, t)$, and Hölder's inequality.

Taking, the estimates (4.34)-(4.36) into consideration, we obtain from (4.28)

$$
\left\{\left\|M_{1}^{\prime} \phi\right\|_{p, Q_{T}}^{(2)}\right\}^{p} \leq \mathcal{K}_{12}\left\{T^{\frac{1}{4}\left(2-\frac{n+2}{p}\right)}+\lambda^{\delta_{0}}\right\}^{p} \cdot \sum_{k}\left\{\left|v_{k}\right|_{p, Q_{T}^{(k)}}^{(4)}\right\}^{p} .
$$

From this inequality and (4.19), (4.20) we get the estimate

$$
\begin{aligned}
\left\{\left\|M_{1}^{\prime} \phi\right\|_{p, Q_{T}}^{(2)}\right\}^{p} \leq & \mathcal{K}_{13}\left\{T^{\frac{1}{4}\left(2-\frac{n+2}{p}\right)}+\lambda^{\delta_{0}}\right\}^{p} \\
& \cdot \sum_{k}\left\{\left\|\eta_{k} f\right\|_{p, Q_{T}^{(k)}}^{(2)}+\left\|\eta_{k} g\right\|_{p, S_{T}^{(k)}}^{\left(3-\frac{1}{p}\right)}\right\}^{p},
\end{aligned}
$$

where $S_{T}^{(k)}=\left\{B\left(x_{k}, \lambda\right) \times(0, T)\right\} \cap S_{T}$. We note that $\mathcal{K}_{13}$ depends on the same parameters as $\mathcal{K}_{1}, \mathcal{K}_{2}$ in Theorem 4.1. In particular, $\mathcal{K}_{13}$ depends on $\bar{T}$, but not on $T$ itself.

We evaluate the right-hand side of (4.38) by using the estimates

$$
\begin{aligned}
& \left\|\mathcal{D}^{\alpha} f\right\|_{p, Q_{T}^{(k)}}^{(2)} \leq \mathcal{K}_{14} T^{1-\frac{|\alpha|}{2}}|f|_{p, Q_{T}^{(k)}}^{(2)}, \quad \text { for }|\alpha|<2, \\
& \left\|\left(\frac{\partial}{\partial t}\right)^{s} \mathcal{D}_{y^{\prime}}^{\gamma} g^{(i)}\right\|_{p, \mathcal{D}_{T}^{(i, k)}} \leq \mathcal{K}_{14} T^{\frac{1}{2}\left(3-j-\frac{1}{p}\right)}\|g\|_{p, S_{T}^{(k)}}^{\left(3-\frac{1}{p}\right)}, \quad \text { for } 2 s+|\alpha|=j \leq 2
\end{aligned}
$$

and

$$
\left|g^{(i)}\right|_{p, \mathcal{D}_{T}^{(i, k)}}^{(l)} \leq \mathcal{K}_{14} T^{\frac{1}{2}\left(3-l-\frac{1}{p}\right)}\|g\|_{p, S_{T}^{(k)}}^{\left(3-\frac{1}{p}\right)}, \quad \text { for } 0 \leq[l]<l<3-\frac{1}{p} .
$$

Here, $[\cdot]$ is the greatest integer function, $\gamma=\left(\gamma_{1}, \ldots, \gamma_{n-1}\right), g^{(i)}\left(y^{\prime}, t\right)$ is the value of the function $g(x, t)$ in the local coordinate system corresponding to the set $\mathcal{U}_{i}, i=i(k)$, and the set $\mathcal{D}_{T}^{(i, k)}$ corresponds to $S_{T}^{(k)}$ after transformation to the local coordinates. The inequalities (4.39), (4.41) follow from Lemma 2.2 and Poincaré's inequality.

Using (4.40), (4.41) and the condition on $T$, we obtain the estimate

$$
\sum_{k}\left\{\left\|\eta_{k} f\right\|_{p, Q_{T}^{(k)}}^{(2)}+\left\|\eta_{k} g\right\|_{p, S_{T}^{(k)}}^{\left(3-\frac{1}{)}\right)}\right\}^{p} \leq \mathcal{K}_{15}\left\{\|f\|_{p, Q_{T}}^{(2)}+\|g\|_{p, S_{T}}^{\left(3-\frac{1}{p}\right)}\right\}^{p}
$$

and, consequently, from (4.38) and (4.42),

$$
\left\|M_{1}^{\prime} \phi\right\|_{p, Q_{T}}^{(2)} \leq \mathcal{K}_{16}\left(T^{\frac{1}{4}\left(2-\frac{n+2}{p}\right)}+\lambda^{\delta_{0}}\right)\left\{\|f\|_{p, Q_{T}}^{(2)}+\|g\|_{p, S_{T}}^{\left(3-\frac{1}{p}\right)}\right\} .
$$


As in (4.28), we establish the following estimate for $M_{1}^{\prime \prime} \phi$ :

$$
\left\{\left\|M_{1}^{\prime \prime} \phi\right\|_{p, Q_{T}}^{(2)}\right\}^{p} \leq \mathcal{K}_{17} \sum_{k}\left\{I_{k}^{(4)}+I_{k}^{(5)}+\sum_{\left|\beta^{\prime}+\beta^{\prime \prime}+\beta^{\prime \prime \prime}\right| \leq 2} I_{k}^{(6)}\left(\beta^{\prime}, \beta^{\prime \prime}, \beta^{\prime \prime \prime}\right)\right\}
$$

where

$$
\begin{array}{r}
I_{k}^{(4)}=\sum_{\alpha^{\prime}, \alpha^{\prime \prime}} \int_{Q_{T}^{(k)}}\left[\left|\mathcal{D}^{\alpha^{\prime}} \xi_{k}(x)\right| \cdot\left|\frac{\partial a_{\alpha}(x, t)}{\partial t}\right| \cdot\left|\mathcal{D}^{\alpha^{\prime \prime}} v_{k}(x, t)\right|\right]^{p} d x d t \\
I_{k}^{(5)}=\sum_{\alpha^{\prime}, \alpha^{\prime \prime}} \int_{Q_{T}^{(k)}}\left[\left|\mathcal{D}^{\alpha^{\prime}} \xi_{k}(x)\right| \cdot\left|a_{\alpha}(x, t)\right| \cdot\left|\frac{\partial}{\partial t} \mathcal{D}^{\alpha^{\prime \prime}} v_{k}(x, t)\right|\right]^{p} d x d t \\
I_{k}^{(6)}\left(\beta^{\prime}, \beta^{\prime \prime}, \beta^{\prime \prime \prime}\right)=\sum_{\alpha^{\prime}, \alpha^{\prime \prime}} \int_{Q_{T}^{(k)}}\left[\left|\mathcal{D}^{\alpha^{\prime}+\beta^{\prime}} \xi_{k}(x)\right| \cdot\left|\mathcal{D}^{\beta^{\prime \prime}} a_{\alpha}(x, t)\right|\right. \\
\left.\cdot\left|\mathcal{D}^{\alpha^{\prime \prime}+\beta^{\prime \prime \prime}} v_{k}(x, t)\right|\right]^{p} d x d t
\end{array}
$$

where the sums are over all $\alpha^{\prime}, \alpha^{\prime \prime}$ such that $\alpha^{\prime}+\alpha^{\prime \prime}=\alpha,|\alpha|=2, \alpha^{\prime}>0$.

Using the boundedness of $a_{\alpha}(x, t)$ and the inequality (4.30), we obtain

$$
I_{k}^{(5)}+\sum_{\left|\beta^{\prime}+\beta^{\prime \prime \prime}\right| \leq 2} I_{k}^{(6)}\left(\beta^{\prime}, 0, \beta^{\prime \prime \prime}\right) \leq \mathcal{K}_{18} \sqrt{\kappa}\left\{\left|v_{k}\right|_{p, Q_{T}^{(k)}}^{(4)}\right\}^{p} .
$$

The estimates for $I_{k}^{(4)}, I_{k}^{(6)}\left(\beta^{\prime}, \beta^{\prime \prime}, \beta^{\prime \prime \prime}\right), \beta^{\prime} \neq 0$, are similar to those for $I_{k}^{(1)}$, $I_{k}^{(3)}\left(\beta^{\prime}, \beta^{\prime \prime}, \beta^{\prime \prime \prime}\right), \beta^{\prime} \neq 0$, respectively, which were obtained in (4.34) and (4.36). From these estimates and (4.19), (4.42) we establish the inequality

$$
\left\|M_{1}^{\prime \prime} \phi\right\|_{p, Q_{T}}^{(2)} \leq \mathcal{K}_{19}\left\{\sqrt{\kappa}+T^{\frac{1}{4}\left(2-\frac{n+2}{p}\right)}\right\}\left\{\|f\|_{p, Q_{T}}^{(2)}+\|g\|_{p, S_{T}}^{\left(3-\frac{1}{p}\right)}\right\} .
$$

Now, we pass on to the evaluation of the principal summands in the representation of $M_{2} \phi$ :

$$
\begin{gathered}
M_{2}^{\prime} \phi=\sum_{k} \sum_{|\beta|=1} \xi_{k}(x)\left[b_{\beta}(x, t)-b_{\beta}\left(x_{k}, 0\right)\right] \mathcal{D}^{\beta} v_{k}(x, t), \\
M_{2}^{\prime \prime} \phi=\sum_{k} \sum_{|\beta|=1} \mathcal{D}^{\beta} \xi_{k}(x) \cdot b_{\beta}(x, t) \cdot v_{k}(x, t) .
\end{gathered}
$$


We consider the evaluation of some typical terms corresponding to the norms of these functions in $W_{p}^{\left(3-\frac{1}{p}\right), 0}\left(S_{T}\right)$ :

$$
\begin{aligned}
J_{k}^{(1)}= & \int_{0}^{T} d t \int_{\Gamma^{(i, k)}} d y^{\prime} \int_{\Gamma^{(i, k)}}\left|\xi_{k}^{(i)}\left(y^{\prime}\right) \cdot\left[b_{\beta}^{(i)}\left(y^{\prime}, t\right)-b_{\beta}^{(i)}\left(z^{\prime}, t\right)\right] \frac{\partial}{\partial t} \mathcal{D}_{y^{\prime}}^{\gamma} v_{k}^{(i)}\left(y^{\prime}, t\right)\right|{ }^{p} \\
& \cdot \frac{d z^{\prime}}{\left|y^{\prime}-z^{\prime}\right|^{n-2+p}}, \\
J_{k}^{(2)}= & \int_{0}^{T} d t \int_{\Gamma^{(i, k)}} d y^{\prime} \int_{\Gamma^{(i, k)}}\left|\xi_{k}^{(i)}\left(y^{\prime}\right) \cdot\left[\frac{\partial}{\partial t} b_{\beta}^{(i)}\left(y^{\prime}, t\right)-\frac{\partial}{\partial t} b_{\beta}^{(i)}\left(z^{\prime}, t\right)\right] \mathcal{D}_{y^{\prime}}^{\gamma} v_{k}^{(i)}\left(y^{\prime}, t\right)\right|^{p} \\
& \cdot \frac{d z^{\prime}}{\left|y^{\prime}-z^{\prime}\right|^{n-2+p}}, \\
J_{k}^{(3)}= & \int_{0}^{T} d t \int_{\Gamma^{(i, k)}} d y^{\prime} \int_{\Gamma^{(i, k)}} \mid \xi_{k}\left(y^{\prime}\right) \cdot\left[b_{\beta}^{(i)}\left(y^{\prime}, t\right)-b_{\beta}^{(i)}\left(y^{\prime}, 0\right)\right] \\
& \left.\cdot\left[\frac{\partial}{\partial t} \mathcal{D}_{y^{\prime}}^{\gamma} v_{k}^{(i)}\left(y^{\prime}, t\right)-\frac{\partial}{\partial t} \mathcal{D}_{z^{\prime}}^{\gamma} v_{k}^{(i)}\left(z^{\prime}, t\right)\right]\right|^{p} \frac{d z^{\prime}}{\left|y^{\prime}-z^{\prime}\right|^{n-2+p}}, \\
J_{k}^{(4)}= & \int_{0}^{T} d t \int_{\Gamma^{(i, k)}} d y^{\prime} \int_{\Gamma^{(i, k)}}\left|\mathcal{D}_{y^{\prime}}^{\gamma} \xi_{k}^{(i)}\left(y^{\prime}\right) b_{\beta}^{(i)}\left(y^{\prime}, t\right) \cdot\left[\mathcal{D}_{y^{\prime}}^{\delta} v_{k}^{(i)}\left(y^{\prime}, t\right)-\mathcal{D}_{z^{\prime}}^{\delta} v_{k}^{(i)}\left(z^{\prime}, t\right)\right]\right|^{p} \\
& \cdot \frac{d z^{\prime}}{\left|y^{\prime}-z^{\prime}\right|^{n-2+p}},
\end{aligned}
$$

where $|\gamma|=1,|\delta|=2, \gamma=\left(\gamma_{1}, \ldots, \gamma_{n-1}\right), \delta=\left(\delta_{1}, \ldots, \delta_{n-1}\right)$, and the set $\Gamma^{(i, k)}$ and the point $y^{\prime}$ correspond to $\partial \Omega \cap B\left(x_{k}, \lambda\right)$ and $x$ in local coordinates, respectively. The function $g^{(i)}\left(y^{\prime}, t\right)$ is the function $g(x, t)$ on $S_{T}^{(k)}$ in terms of local coordinates.

For the evaluation of the integrals from (4.50) we shall use the following estimates:

$$
\left\|\left(\frac{\partial}{\partial t}\right)^{s} \mathcal{D}_{y^{\prime}}^{\alpha} v_{k}^{(i)}\right\|_{p, \Gamma(i, k)} \leq \mathcal{K}_{20} T^{\frac{1}{2}\left(4-j-\frac{1}{p}\right)}\left\|v_{k}\right\|_{p, S_{T}^{(k)}}^{\left(4-\frac{1}{)}\right)}
$$

for $2 s+|\alpha|=j \leq 3$,

$$
\left|v_{k}^{(i)}\right|_{p, \Gamma^{(i, k)}}^{(j)} \leq \mathcal{K}_{20} T^{\frac{1}{2}\left(4-j-\frac{1}{p}\right)}\left\|v_{k}\right\|_{p, S_{T}^{(k)}}^{\left(4-\frac{1}{)}\right)}
$$

for a non-integer and positive $j$ which satisfies the condition $j<4-\frac{1}{p}$, and

$$
\left|v_{k}\right|_{S_{T}^{(k)}}^{(j)} \leq \mathcal{K}_{20} T^{\frac{1}{2}\left(4-j-\frac{n+2}{p}\right)}\left\|v_{k}\right\|_{p, S_{T}^{(k)}}^{\left(4-\frac{1}{)}\right)}
$$

for $0 \leq j<4-\frac{n+2}{p}$. The inequalities (4.51)-(4.53) follow from Lemma 2.2 and Poincaré's inequality. From $\left.b_{1}\right)$ and Lemma 2.2 it follows that $b_{\beta} \in \mathcal{C}^{(j)}\left(S_{T}\right)$ for $j<3-\frac{n+2}{p}$. Then by using (4.51) and a simple calculation we obtain the inequality

$$
J_{k}^{(1)}+J_{k}^{(3)} \leq K_{21} \lambda^{p}\left\{\left\|v_{k}\right\|_{p, S_{T}^{(k)}}^{\left(4-\frac{1}{p}\right)}\right\}^{p} .
$$


The next estimates are obtained by using the inequalities (4.52), (4.53) and Condition $\left.b_{1}\right)$ :

$$
\begin{aligned}
& J_{k}^{(2)} \leq \mathcal{K}_{22} T^{\frac{p}{2}\left(3-\frac{n+2}{p}\right)}\left\{\left\|v_{k}\right\|_{p, S_{T}^{(k)}}^{\left(4-\frac{1}{p}\right)}\right\}^{p}, \\
& J_{k}^{(4)} \leq \mathcal{K}_{22} \kappa^{\frac{p}{2}}\left\{\left\|v_{k}\right\|_{p, S_{T}^{(k)}}^{\left(4-\frac{1}{p}\right)}\right\}^{p} .
\end{aligned}
$$

Evaluating in an analogous way, we establish the following estimates by using the inequalities (4.19), (4.42):

$$
\begin{aligned}
& \left\|M_{2}^{\prime} \phi\right\|_{p, S_{T}}^{\left(3-\frac{1}{p}\right)} \leq K_{23}\left\{\lambda+T^{\frac{1}{2}\left(2-\frac{n+2}{p}\right)}\right\}\left\{\|f\|_{p, Q_{T}}^{(2)}+\|g\|_{p, S_{T}}^{\left(3-\frac{1}{p}\right)}\right\}, \\
& \left\|M_{2}^{\prime \prime} \phi\right\|_{p, S_{T}}^{\left(3-\frac{1}{p}\right)} \leq \mathcal{K}_{23}\left\{\sqrt{\kappa}+T^{\frac{1}{2}\left(2-\frac{n+2}{p}\right)}\right\}\left\{\|f\|_{p, Q_{T}}^{(2)}+\|g\|_{p, S_{T}}^{\left(3-\frac{1}{p}\right)}\right\} .
\end{aligned}
$$

The inequality (4.25) now follows by a suitable choice of $\lambda_{1}, \kappa_{1}$ from (4.24), (4.43), (4.47), (4.56) and the analogous estimates for lower terms in $M_{1} \phi, M_{2} \phi$. This completes the proof.

Let us define an operator $N: W_{p}^{(4), 0}\left(Q_{T}\right) \rightarrow W_{p}^{(4), 0}\left(Q_{T}\right)$ by

$$
N u=\sum_{k} \xi_{k} R^{(k)}\left(\eta_{k} L u-L\left(\eta_{k} u\right), \eta_{k} B u-B\left(\eta_{k} u\right)\right) .
$$

Lemma 4.2. Assume that the conditions of Theorem 4.1 are satisfied. Then there exist positive numbers $\lambda_{2}, \kappa_{2}$, depending on the same parameters as $\mathcal{K}_{1}, \mathcal{K}_{2}$ in Theorem 4.1, such that for

$$
T=\lambda^{2} \kappa, \quad 0<\lambda \leq \lambda_{2}, \quad 0<\kappa \leq \min \left\{1, \kappa_{2}\right\},
$$

we have

$$
\|N u\|_{p, Q_{T}}^{(4)} \leq \frac{1}{2}\|u\|_{p, Q_{T}}^{(4)} .
$$

Proof. We choose $T=\lambda^{2} \kappa$ with $\kappa \leq 1$. The summands in

$$
\begin{aligned}
\eta_{k}(x) L u-L\left(\eta_{k} u\right) & =\sum_{\substack{|\alpha| \leq 2 \\
\alpha^{\prime}+\alpha^{\prime \prime}=\alpha, \alpha^{\prime}>0}} a_{\alpha}(x) \mathcal{D}^{\alpha^{\prime}} \eta_{k}(x) \mathcal{D}^{\alpha^{\prime \prime}} u(x), \\
\eta_{k}(x) B u-B\left(\eta_{k} u\right)= & \sum_{|\beta|=1} b_{\beta}(x) \mathcal{D}^{\beta} \eta_{k}(x) u(x)
\end{aligned}
$$

are analogous to the summands in $M_{1}^{\prime \prime} \phi, M_{2}^{\prime \prime} \phi$, respectively. In a manner analogous to that of the proof of the estimates (4.47), (4.56), we obtain the inequalities

$$
\begin{gathered}
\left\|\eta_{k} L u-L\left(\eta_{k} u\right)\right\|_{p, Q_{T}}^{(2)} \leq \mathcal{K}_{24}\left\{\sqrt{\kappa}+T^{\frac{1}{4}\left(2-\frac{n+2}{p}\right)}\right\}\left\|\zeta_{k} u\right\|_{p, Q_{T}}^{(4)} \\
\left\|\eta_{k} B u-B\left(\eta_{k} u\right)\right\|_{p, S_{T}}^{\left(3-\frac{1}{p}\right)} \leq \mathcal{K}_{24}\left\{\sqrt{\kappa}+T^{\frac{1}{2}\left(2-\frac{n+2}{p}\right)}\right\}\left\|\zeta_{k} u\right\|_{p, Q_{T}}^{(4)} .
\end{gathered}
$$

Using the a priori estimate (4.19), the inequalities (4.30), (4.60), (4.61) and the choice of $T$, we establish the estimate

$$
\|N u\|_{p, Q_{T}}^{(4)} \leq \mathcal{K}_{25}\left\{\sqrt{\kappa}+T^{\frac{1}{4}\left(2-\frac{n+2}{p}\right)}\right\} \sum_{k}\left\|\zeta_{k}\right\|_{p, Q_{T}}^{(4)}
$$


The last sum can be evaluated by the inequality

$$
\sum_{k}\left\|\zeta_{k} u\right\|_{p, Q_{T}}^{(4)} \leq \mathcal{K}_{26}\|u\|_{p, Q_{T}}^{(4)}
$$

which follows from (4.15) and (4.30).

We now obtain the estimate (4.59) from (4.62) and (4.63) by suitable choice of $\lambda_{1}, \kappa_{2}$.

Proof of Theorem 4.1, Continued. Let us fix the numbers $\lambda_{0}=\min \left\{\lambda_{1}, \lambda_{2}\right\}, \kappa_{0}=$ $\min \left\{1, \kappa_{1}, \kappa_{2}\right\}$ and $T^{(0)}=\lambda_{0}^{2} \kappa_{0}$ so that inequalities (4.25) and (4.59) are satisfied. By the contraction mapping principle, we can conclude that the operators $I+M, I+N$ have bounded inverses $(I+M)^{-1},(I+N)^{-1}$ defined on the spaces $W_{p}^{\left(2,3-\frac{3}{p}\right), 0}\left(Q_{T}, S_{T}\right), W_{p}^{(4), 0}\left(Q_{T}\right)$, respectively. Applying the operator $(I+N)^{-1}$ to the second equality in (4.11) and replacing $\phi$ in the first of (4.11) by $(I+M)^{-1} \psi$, we obtain, for any $\psi \in W_{p}^{\left(2,3-\frac{3}{p}\right), 0}\left(Q_{T}, S_{T}\right)$ and $v \in W_{p}^{(4), 0}\left(Q_{T}\right)$,

$$
P R(1+M)^{-1} \psi=\psi, \quad(I+N)^{-1} R P v=v .
$$

This means that the operator $P$ has a bounded inverse, and completes the proof of the assertion of the theorem for $T=T^{(0)}$.

Taking into consideration the fact that $T$ depends only on the same parameters as the constants $\mathcal{K}_{1}, \mathcal{K}_{2}$, we can apply the result just proved successively on the intervals $\left(j \frac{T^{(0)}}{2},(j+2) \frac{T^{(0)}}{2}\right), k=0,1, \ldots$ Using the reduction of the initialboundary value problem to the problem with zero initial data, we can complete the proof of the theorem in the standard way.

\section{Proof of Theorems 3.1 And 3.2}

In order to further study the properties of the operator $A$, defined in (3.18), we need the following four auxiliary lemmas. We always assume that the number $p$ satisfies condition (2.11), and that $\Omega$ is a bounded open set in $\mathcal{R}^{n}$ with $\partial \Omega$ of class $\mathcal{C}^{4}$.

Lemma 5.1. Let $\Phi(x, t, v)$ be a function defined for $(x, t) \in Q_{T}$ and the vector $v=\left(v_{1}, v_{2}, \ldots, v_{M}\right) \in \mathcal{R}^{M}$. Assume that $\Phi(x, t, v)$ is a continuous function and that the operator $\widetilde{\Phi}$, defined by

$$
[\widetilde{\Phi}(v)](x, t)=\Phi(x, t, v(x, t))
$$

is bounded and continuous acting from $\left[W_{p}^{(2), 0}\left(Q_{T}\right)\right]^{M}$ to $W_{p}^{(2)}\left(Q_{T}\right)$. Then for arbitrary sequences $v^{(j)}(x, t)$ (resp. $\left.w_{j}(x, t)\right), j=1,2, \ldots$, weakly convergent in the space $\left[W_{p}^{(2), 0}\left(Q_{T}\right)\right]^{M}\left(\right.$ resp. $\left.W_{p}^{(2), 0}\left(Q_{T}\right)\right)$ to $v^{(0)}(x, t)$ (resp. $\left.w_{0}(x, t)\right)$, the sequence $\rho_{j}$, defined by

$$
\rho_{j}(x, t)=\left\{\left[\widetilde{\Phi}\left(v^{(j)}\right)\right](x, t)-\left[\widetilde{\Phi}\left(v^{(0)}\right)\right](x, t)\right\}\left(w_{j}(x, t)-w_{0}(x, t)\right),
$$

converges strongly to zero in $W_{p}^{(2), 0}\left(Q_{T}\right)$. 
Proof. By the compactness of the embedding of $W_{p}^{1}\left(Q_{T}\right)$ in $L_{p}\left(Q_{T}\right)$ and Lemma 2.2 we obtain the strong convergence of the sequences $v^{(j)}(x, t), w_{j}(x, t)$ in the spaces $\left[\mathcal{C}^{(\delta)}\left(\overline{Q_{T}}\right)\right]^{M}, \mathcal{C}^{(\delta)}\left(\overline{Q_{T}}\right)$, respectively, for $0<\delta<2-\frac{n+2}{p}$. Thus, from the continuity of the function $\Phi(x, t, v)$ we immediately obtain the strong convergence of the sequence $\rho_{j}(x, t)$ to zero in $L_{p}\left(Q_{T}\right)$ and the uniform convergence to zero of the sequence

$$
\rho_{j}^{\prime}(x, t)=\left[\widetilde{\Phi}\left(v^{(j)}\right](x, t)-\left[\widetilde{\Phi}\left(v^{(0)}\right)\right](x, t)\right.
$$

In the relation

$$
\frac{\partial}{\partial t} \rho_{j}(x, t)=\frac{\partial}{\partial t} \rho_{j}^{\prime}(x, t)\left[w_{j}(x, t)-w_{0}(x, t)\right]+\frac{\partial}{\partial t}\left[w_{j}(x, t)-w_{0}(x, t)\right] \rho_{j}^{\prime}(x, t)
$$

both summands on the right-hand side tend to zero in $L_{p}\left(Q_{T}\right)$, since their first factors are bounded in $L_{p}\left(Q_{T}\right)$ and their second are uniformly convergent to zero. Analogously, we can verify the strong convergence in $L_{p}\left(Q_{T}\right)$ of the derivatives $\mathcal{D}^{\alpha} \rho_{j}(x, t)$ with $|\alpha|=1$.

For the second order derivatives with respect to $x$ we have

$$
\begin{aligned}
\mathcal{D}^{\alpha} \rho_{j}(x, t)= & \mathcal{D}^{\alpha} \rho_{j}^{\prime}(x, t)\left[w_{j}(x, t)-w_{0}(x, t)\right] \\
& +\left\{\mathcal{D}^{\alpha}\left[w_{j}(x, t)-w_{0}(x, t)\right]\right\} \rho_{j}^{\prime}(x, t) \\
& +2 \sum_{\substack{\left|\alpha^{\prime}\right|=\left|\alpha^{\prime \prime}\right|=1 \\
\alpha^{\prime}+\alpha^{\prime \prime}=\alpha}} \mathcal{D}^{\alpha^{\prime}} \rho_{j}^{\prime}(x, t) \mathcal{D}^{\alpha^{\prime \prime}}\left[w_{j}(x, t)-w_{0}(x, t)\right] .
\end{aligned}
$$

The strong convergence to zero in $L_{p}\left(Q_{T}\right)$ of the first and second terms on the righthand side of (5.4) follows in the same way as for the terms in (5.3). By Lemma 2.3 we obtain the strong convergence, in $L_{2 p}\left(Q_{T}\right)$, to zero of the sequences $\mathcal{D}^{\alpha^{\prime}} \rho_{j}^{\prime}(x, t)$ and $\mathcal{D}^{\alpha^{\prime \prime}}\left[w_{j}(x, t)-w_{0}(x, t)\right]$ for $\left|\alpha^{\prime}\right|=\left|\alpha^{\prime \prime}\right|=1$. This guarantees the strong convergence in $L_{p}\left(Q_{T}\right)$ of the last summand on the right-hand side of (5.4). The proof of Lemma 5.1 is complete.

Lemma 5.2. Assume that the conditions of Theorem 3.1 are satisfied and let $u_{j}$ be a sequence in $W_{p}^{(4), 0}\left(Q_{T}\right)$ which converges weakly to some function $u_{0}$. Then the sequence $r_{j}^{(1)}$, defined by

$$
r_{j}^{(1)}(x, t)=F\left(x, t, u_{j}, \mathcal{D}^{1} u_{j}, \mathcal{D}^{2} u_{j}\right)-F\left(x, t, \mathcal{D}^{1} u_{0}, \mathcal{D}^{2} u_{0}\right)-L\left(u_{0}\right)\left(u_{j}-u_{0}\right),
$$

converges strongly to zero in $W_{p}^{(2), 0}\left(Q_{T}\right)$, where the operator $L\left(u_{0}\right)$ is defined in (3.5).

Proof. We rewrite $r_{j}^{(1)}(x, t)$ as follows:

$$
\begin{aligned}
r_{j}^{(1)}(x, t)= & \sum_{|\alpha| \leq 2} \int_{0}^{1}\left\{F _ { \alpha } \left(x, t, u_{j}^{(s)}, \mathcal{D}^{1} u_{j}^{(s)}, \mathcal{D}^{2} u_{j}^{(s))}\right.\right. \\
& \left.-F_{\alpha}\left(x, t, \mathcal{D}^{1} u_{0}, \mathcal{D}^{2} u_{0}\right)\right\} d s \cdot \mathcal{D}^{\alpha}\left[u_{j}(x, t)-u_{0}(x, t)\right],
\end{aligned}
$$

where $u_{j}^{(s)}(x, t)=s u_{j}(x, t)+(1-s) u_{0}(x, t)$ and $F_{\alpha}(x, t, \xi)$ is defined in Condition $\left.F_{2}\right)$. Now, the strong convergence of the sequence $r_{j}^{(1)}$ follows immediately from Conditions $F_{3}$ ), $F_{4}$ ) and Lemma 5.1. 
Lemma 5.3. Let $\Psi(x, t, v)$ be defined for $(x, t) \in Q_{T}, v=\left(v_{1}, \ldots, v_{M}\right) \in \mathcal{R}^{M}$. Assume that $\Psi(x, t, v)$ is continuous and that the operator $\widetilde{\Psi}$, defined by

$$
[\widetilde{\Psi}(v)](x, t)=\Psi(x, t, v(x, t))
$$

is bounded and continuous acting from $\left[W_{p}^{\left(3-\frac{1}{p}\right), 0}\left(S_{T}\right)\right]^{M}$ to $W_{p}^{\left(3-\frac{1}{p}\right)}\left(S_{T}\right)$. Then for any sequences $v^{(j)}\left(\right.$ resp. $\left.w_{j}\right), j=1,2, \ldots$, weakly convergent in $\left[W_{p}^{\left(3-\frac{1}{p}\right), 0}\left(S_{T}\right)\right]^{M}$ $\left(\right.$ resp. $\left.W_{p}^{\left(3-\frac{1}{p}\right), 0}\left(S_{T}\right)\right)$ to $v^{(0)}\left(\right.$ resp. $\left.w_{0}\right)$, the sequence $\sigma_{j}$, defined by

$$
\sigma_{j}(x, t)=\left\{\left[\widetilde{\Psi}\left(v^{(j)}\right)\right](x, t)-\left[\widetilde{\Psi}\left(v^{(0)}\right)\right](x, t)\right\}\left(w_{j}(x, t)-w_{0}(x, t)\right),
$$

converges strongly to zero in $W_{p}^{\left(3-\frac{1}{p}\right), 0}\left(S_{T}\right)$.

Proof. The strong convergence of the sequence $\sigma_{j}(x, t)$ in $W_{p}^{(2)}\left(S_{T}\right)$ follows as in the proof of Lemma 5.1. We need to show that $\left|\sigma_{j}(x, t)\right|_{p, S_{T}}^{\left(3-\frac{1}{p}\right)}$ converges to zero. To this end, we show the convergence to zero of the following typical term:

$$
\begin{array}{r}
I_{j}^{(i)}=\int_{0}^{T} d t \int_{B^{\prime}(d)} d y^{\prime} \int_{B^{\prime}(d)}\left|\frac{\partial}{\partial t} \sigma_{j}^{(i)}\left(y^{\prime}, t\right)-\frac{\partial}{\partial t} \sigma_{j}^{(i)}\left(z^{\prime}, t\right)\right|^{p} \\
\cdot \frac{d z^{\prime}}{\left|y^{\prime}-z^{\prime}\right|^{n+p-2}},
\end{array}
$$

where $\sigma_{j}^{(i)}\left(y^{\prime}, t\right)$ is the value of the function $\sigma_{j}(x, t)$ in the local coordinate system at the point $\xi_{i}$. The index ( $i$ ) below has an analogous meaning.

From Lemma 2.2 we obtain the strong convergence of the following sequences:

$$
\begin{gathered}
\sigma_{j}^{\prime}(x, t)=\left[\widetilde{\Psi}\left(v^{(j)}\right)\right](x, t)-\left[\widetilde{\Psi}\left(v^{(0)}\right)\right](x, t) \rightarrow 0 \quad \text { in } \mathcal{C}^{(1+\delta)}\left(S_{T}\right), \\
w_{j}^{\prime}(x, t)=w_{j}(x, t)-w_{0}(x, t) \rightarrow 0 \quad \text { in } \mathcal{C}^{(1+\delta)}\left(S_{T}\right),
\end{gathered}
$$

for $0<\delta<2-\frac{n+2}{p}$. Using the estimate

$$
\begin{aligned}
& \left|\frac{\partial}{\partial t} \sigma_{j}^{(i)}\left(y^{\prime}, t\right)-\frac{\partial}{\partial t} \sigma_{j}^{(i)}\left(z^{\prime}, t\right)\right| \\
& \leq\left|\frac{\partial}{\partial t}\left(\sigma_{j}^{\prime}\right)^{(i)}\left(y^{\prime}, t\right)-\frac{\partial}{\partial t}\left(\sigma_{j}^{\prime}\right)^{(i)}\left(z^{\prime}, t\right)\right| \cdot\left|\left(w_{j}^{\prime}\right)^{(i)}\left(z^{\prime}, t\right)\right| \\
& \quad+\left|\frac{\partial}{\partial t}\left(w_{j}^{\prime}\right)^{(i)}\left(y^{\prime}, t\right)-\frac{\partial}{\partial t}\left(w_{j}^{\prime}\right)^{(i)}\left(z^{\prime}, t\right)\right| \cdot\left|\left(\sigma_{j}^{\prime}\right)^{(i)}\left(z^{\prime}, t\right)\right| \\
& \quad+\left[\left|\frac{\partial}{\partial t}\left(\sigma_{j}^{\prime}\right)^{(i)}\left(y^{\prime}, t\right)\right| \cdot\left|w_{j}^{\prime}\right|_{S_{T}}^{(1+\delta)}+\left|\frac{\partial}{\partial t}\left(w_{j}^{\prime}\right)^{(i)}\left(y^{\prime}, t\right)\right| \cdot\left|\sigma_{j}^{\prime}\right|_{S_{T}}^{(1+\delta)}\right] \cdot\left|y^{\prime}-z^{\prime}\right|
\end{aligned}
$$

and the convergence in (5.9) and (5.10), it is simple to check that $I_{j}^{(i)}$ tends to zero as $j$ tends to infinity. In an analogous way we can evaluate the other summands in the norm of the function $\sigma_{j}(x, t)$ in $W_{p}^{\left(3-\frac{1}{p}\right), 0}\left(S_{T}\right)$. The proof of the lemma is complete. 
Lemma 5.4. Assume that the conditions of Theorem 3.1 are satisfied, and let $u_{j}$ be an arbitrary sequence in $W_{p}^{(4), 0}\left(Q_{T}\right)$ which converges weakly to some function $u_{0}$. Then the sequence

$$
r_{j}^{(2)}(x, t)=G\left(x, t, u_{j}, \mathcal{D}^{1} u_{j}\right)-G\left(x, t, u_{0}, \mathcal{D}^{1} u_{0}\right)-B\left(u_{0}\right)\left(u_{j}-u_{0}\right)
$$

converges strongly to zero in $W_{p}^{\left(3-\frac{1}{p}\right), 0}\left(S_{T}\right)$, where the operator $B\left(u_{0}\right)$ is defined in (3.6).

Proof. We rewrite $r_{j}^{(2)}(x, t)$ as follows:

$$
r_{j}^{(2)}(x, t)=\sum_{|\beta| \leq 1} \int_{0}^{1}\left\{G_{\beta}\left(x, t, u_{j}^{(s)}, \mathcal{D}^{1} u_{j}^{(s)}-G_{\beta}\left(x, t, u_{0}, \mathcal{D}^{1} u_{0}\right)\right\} \cdot \mathcal{D}^{\beta}\left(u_{j}-u_{0}\right) d s\right.
$$

where $u_{j}^{(s)}(x, t)=s u_{j}(x, t)+(1-s) u_{0}(x, t)$. The strong convergence of $r_{j}^{(2)}(x, t)$ follows immediately from Lemma 5.3 and the properties of traces of functions from the space $W_{p}^{(4), 0}\left(Q_{T}\right)$ which were formulated in Lemma 2.1.

Proof of Theorem 3.1. The boundedness of the operator $A$, defined in (3.18), follows from the estimates (3.10) and (3.17). Using (3.9), Conditions $\left.F_{4}\right), G_{4}$ ) and the embedding theorems, it is simple to show the continuity of $A$.

We are now going to check that the operator $A$ satisfies Condition $(S)_{+}$on the space $W_{p}^{(4), 0}\left(Q_{T}\right)$. To this end, let $u_{j} \in W_{p}^{(4), 0}\left(Q_{T}\right), j=1,2, \ldots$, be such that

$$
u_{j} \rightarrow u_{0}, \quad \limsup _{j \rightarrow \infty}\left\langle A u_{j}, u_{j}-u_{0}\right\rangle \leq 0 .
$$

We define the sequence

$$
E_{j}=\left\langle A_{E}\left(u_{j}, \frac{\partial u_{j}}{\partial t}-F\left(\cdot, \cdot, u_{j}, \mathcal{D}^{1} u_{j}, \mathcal{D}^{2} u_{j}\right)-f\right), u_{j}-u_{0}\right\rangle .
$$

From (3.11) we obtain

$$
\begin{aligned}
E_{j}= & \sum_{2 s+|\alpha| \leq 2} \int_{Q_{T}} \psi_{p}\left\{\left(\frac{\partial}{\partial t}\right)^{s} \mathcal{D}^{\alpha}\left[\frac{\partial u_{j}}{\partial t}-F_{j}(x, t)\right]\right\} \\
& \cdot\left(\frac{\partial}{\partial t}\right)^{s} \mathcal{D}^{\alpha}\left[\frac{\partial}{\partial t}\left(u_{j}-u_{0}\right)-L\left(u_{j}\right)\left(u_{j}-u_{0}\right)\right] d x d t,
\end{aligned}
$$

where $L(u)$ is the operator introduced in (3.5) and $F_{j}(x, t)$ is defined by

$$
F_{j}(x, t)=F\left(x, t, u_{j}, \mathcal{D}^{1} u_{j}, \mathcal{D}^{2} u_{j}\right)-f(x, t), \quad j=0,1,2, \ldots
$$

From Lemmas 5.1 and 5.2 we know that the sequences

$$
\begin{gathered}
F_{j}(x, t)-F_{0}(x, t)-L\left(u_{0}\right)\left(u_{j}-u_{0}\right), \\
L\left(u_{j}\right)\left(u_{j}-u_{0}\right)-L\left(u_{0}\right)\left(u_{j}-u_{0}\right)
\end{gathered}
$$

converge strongly to zero in $W_{p}^{(2), 0}\left(Q_{T}\right)$. The convergence of these sequences implies that

$$
\begin{aligned}
& E_{j}-\sum_{2 s+|\alpha| \leq 2} \int_{Q_{T}} \psi_{p}\left\{\left(\frac{\partial}{\partial t}\right)^{s} \mathcal{D}^{\alpha}\left[\frac{\partial u_{j}}{\partial t}-F_{0}(x, t)-L\left(u_{0}\right)\left(u_{j}-u_{0}\right)\right]\right\} \\
& \cdot\left(\frac{\partial}{\partial t}\right)^{s} \mathcal{D}^{\alpha}\left[\frac{\partial}{\partial t}\left(u_{j}-u_{0}\right)-L\left(u_{0}\right)\left(u_{j}-u_{0}\right)\right] d x d t \rightarrow 0 \quad \text { as } j \rightarrow \infty .
\end{aligned}
$$


Using the weak convergence of $u_{j}(x, t)$ to $u_{0}(x, t)$ in $W_{p}^{(4), 0}\left(Q_{T}\right)$, we obtain

$$
\begin{aligned}
& \lim _{j \rightarrow \infty} \sum_{2 s+|\alpha| \leq 2} \int_{Q_{T}} \psi_{p}\left\{\left(\frac{\partial}{\partial t}\right)^{s} \mathcal{D}^{\alpha}\left[\frac{\partial u_{0}}{\partial t}-F_{0}(x, t)\right]\right\} \\
& \cdot\left(\frac{\partial}{\partial t}\right)^{s} \mathcal{D}^{\alpha}\left[\frac{\partial}{\partial t}\left(u_{j}-u_{0}\right)-L\left(u_{0}\right)\left(u_{j}-u_{0}\right)\right] d x d t=0 .
\end{aligned}
$$

We note that there exists a positive constant $\mathcal{C}_{p}$, depending only on $p$, such that for any numbers $s_{1}, s_{2} \in \mathcal{R}$ the inequality

$$
\left[\psi\left(s_{1}\right)-\psi\left(s_{2}\right)\right]\left(s_{1}-s_{2}\right) \geq \mathcal{C}_{p}\left|s_{1}-s_{2}\right|^{p}
$$

holds for the function $\psi_{p}(s)$ defined in (3.12).

From (5.16)-(5.18) we obtain the estimate

$$
\begin{aligned}
E_{j} & \geq \mathcal{C}_{p} \sum_{2 s+|\alpha| \leq 2} \int_{Q_{T}}\left|\left(\frac{\partial}{\partial t}\right)^{s} \mathcal{D}^{\alpha}\left[\frac{\partial}{\partial t}\left(u_{j}-u_{0}\right)-L\left(u_{0}\right)\left(u_{j}-u_{0}\right)\right]\right|^{p} d x d t+\epsilon_{j}^{\prime} \\
& =\mathcal{C}_{p}\left\{\left\|\frac{\partial}{\partial t}\left(u_{j}-u_{0}\right)-L\left(u_{0}\right)\left(u_{j}-u_{0}\right)\right\|_{p, Q_{T}}^{(2)}\right\}^{p}+\epsilon_{j}^{\prime},
\end{aligned}
$$

where $\epsilon_{j}^{\prime}$ is a sequence that tends to zero as $j \rightarrow \infty$.

Now let us define the sequence $B_{j}$ by

$$
B_{j}=\sum_{k=1}^{3} B_{j}^{(k)}, \quad B_{j}^{(k)}=\left\langle A_{B}^{(k)}\left(u_{j}, G\left(x, t, u_{j}, \mathcal{D}^{1} u_{j}\right)-g\right), u_{j}-u_{0}\right\rangle .
$$

We want to derive the estimate

$$
B_{j} \geq \mathcal{C}_{p}\left\{\left\|B\left(u_{0}\right)\left(u_{j}-u_{0}\right)\right\|_{p, S_{T}}^{\left(3-\frac{1}{p}\right)}\right\}^{p}+\epsilon_{j}^{\prime \prime},
$$

where $\epsilon_{j}^{\prime \prime} \rightarrow 0$ as $j \rightarrow \infty$. The operators $A_{B}^{(k)}, B\left(u_{0}\right)$ were defined in (3.14)-(3.16) and (3.6), respectively.

We are going to evaluate only a typical term in $B_{j}$, namely, $B_{j}^{(2)}$. The same type of argument goes through for $B_{j}^{(1)}$ and $B_{j}^{(3)}$. From (3.15) we obtain

$$
\begin{aligned}
B_{j}^{(2)}= & \sum_{j=1}^{I} \sum_{2 s+|\beta|=2}^{\prime} \int_{0}^{T} d t \int_{B^{\prime}(d)} d y^{\prime} \int_{B^{\prime}(d)} \\
& \cdot \psi_{p}\left\{\left(\frac{\partial}{\partial t}\right)^{s} \mathcal{D}_{y}^{\beta} G_{j}^{(i)}\left(y^{\prime}, t\right)-\left(\frac{\partial}{\partial t}\right)^{s} \mathcal{D}_{z}^{\beta} G_{j}^{(i)}\left(z^{\prime}, t\right)\right\} \\
& \cdot\left\{\left(\frac{\partial}{\partial t}\right)^{s} \mathcal{D}_{y}^{\beta}\left[B\left(u_{j}\right)\left(u_{j}-u_{0}\right)\right]^{(i)}\left(y^{\prime}, t\right)\right. \\
& \left.-\left(\frac{\partial}{\partial t}\right)^{s} \mathcal{D}_{z}^{\beta}\left[B\left(u_{j}\right)\left(u_{j}-u_{0}\right)\right]^{(i)}\left(z^{\prime}, t\right)\right\} \frac{d z^{\prime}}{\left|y^{\prime}-z^{\prime}\right|^{n+p-2}}
\end{aligned}
$$

where the function $G_{j}(x, t)$ is defined by

$$
G_{j}(x, t)=G\left(x, t, u_{j}, \mathcal{D}^{1} u_{j}\right)-g(x, t), \quad(x, t) \in S_{T}, j=0,1, \ldots,
$$

while $G_{j}^{(i)}\left(y^{\prime}, t\right)$ and $\left[B\left(u_{j}\right)\left(u_{j}-u_{0}\right)\right]^{(i)}\left(y^{\prime}, t\right)$ are the values of the functions $G_{j}(x, t)$ and $\left[B\left(u_{j}\right)\left(u_{j}-u_{0}\right)\right](x, t)$, respectively, in local coordinates at the point $\xi_{i}$. 
By Lemmas 5.3 and 5.4 the sequences

$$
\begin{gathered}
G_{j}(x, t)-G_{0}(x, t)-B\left(u_{0}\right)\left(u_{j}-u_{0}\right), \\
B\left(u_{j}\right)\left(u_{j}-u_{0}\right)-B\left(u_{0}\right)\left(u_{j}-u_{0}\right)
\end{gathered}
$$

converge strongly to zero in $W_{p}^{\left(3-\frac{1}{p}\right), 0}\left(S_{T}\right)$. The convergence of these sequences implies

$$
\begin{aligned}
B_{j}^{(2)} & -\sum_{i=1}^{I} \sum_{s s+|\beta|=2}^{\prime} \int_{0}^{T} d t \int_{B^{\prime}(d)} d y^{\prime} \int_{B^{\prime}(d)} \psi_{p}\left\{\left(\frac{\partial}{\partial t}\right)^{s} \mathcal{D}_{y}^{\beta} \widetilde{G}_{j}^{(i)}\left(y^{\prime}, t\right)\right. \\
& \left.-\left(\frac{\partial}{\partial t}\right)^{s} \mathcal{D}_{z}^{\beta} \widetilde{G}_{j}^{(i)}\left(z^{\prime}, t\right)\right\} \cdot\left\{\left(\frac{\partial}{\partial t}\right)^{s} \mathcal{D}_{y}^{\beta}\left[B\left(u_{0}\right)\left(u_{j}-u_{0}\right)\right]^{(i)}\left(y^{\prime}, t\right)\right. \\
& \left.-\left(\frac{\partial}{\partial t}\right)^{s} \mathcal{D}_{z}^{\beta}\left[B\left(u_{0}\right)\left(u_{j}-u_{0}\right)\right]^{(i)}\left(z^{\prime}, t\right)\right\} \frac{d z^{\prime}}{\left|y^{\prime}-z^{\prime}\right|^{n+p-2}} \rightarrow 0
\end{aligned}
$$

as $j \rightarrow \infty$, where we have used the following notation:

$$
\widetilde{G}_{j}(x, t)=G_{0}(x, t)-B\left(u_{0}\right)\left(u_{j}-u_{0}\right) .
$$

Using the weak convergence of $u_{j}$ to $u_{0}$ in $W_{p}^{(4), 0}\left(Q_{T}\right)$, we have

$$
\begin{aligned}
& \sum_{i=1}^{I} \sum_{2 s+|\beta|=2}^{\prime} \int_{0}^{T} d t \int_{B^{\prime}(d)} d y^{\prime} \int_{B^{\prime}(d)} \\
& \cdot \psi_{p}\left\{\left(\frac{\partial}{\partial t}\right)^{s} \mathcal{D}_{y}^{\beta} G_{0}^{(i)}\left(y^{\prime}, t\right)-\left(\frac{\partial}{\partial t}\right)^{s} \mathcal{D}_{z}^{\beta} G_{0}^{(i)}\left(z^{\prime}, t\right)\right\} \\
& \cdot\left\{\left(\frac{\partial}{\partial t}\right)^{s} \mathcal{D}_{y}^{\beta}\left[B\left(u_{0}\right)\left(u_{j}-u_{0}\right)\right]^{(i)}\left(y^{\prime}, t\right)\right. \\
& \left.-\left(\frac{\partial}{\partial t}\right)^{s} \mathcal{D}_{z}^{\beta}\left[B\left(u_{0}\right)\left(u_{j}-u_{0}\right)\right]^{(i)}\left(z^{\prime}, t\right)\right\} \frac{d z^{\prime}}{\left|y^{\prime}-z^{\prime}\right|^{n+p-2}} \rightarrow 0
\end{aligned}
$$

as $j \rightarrow \infty$. From $(5.25),(5.26)$ and $(5.18)$ we get

$$
\begin{aligned}
B_{j}^{(2)} \geq & \mathcal{C}_{p} \sum_{i=1}^{I} \sum_{2 s+|\beta|=2}^{\prime} \int_{0}^{T} d t \int_{B^{\prime}(d)} d y^{\prime} \int_{B^{\prime}(d)} \\
& \cdot \mid\left(\frac{\partial}{\partial t}\right)^{s} \mathcal{D}_{y}^{\beta}\left[B\left(u_{0}\right)\left(u_{j}-u_{0}\right)\right]^{(i)}\left(y^{\prime}, t\right) \\
& -\left.\left(\frac{\partial}{\partial t}\right)^{s} \mathcal{D}_{z}^{\beta}\left[B\left(u_{0}\right)\left(u_{j}-u_{0}\right)\right]^{(i)}\left(z^{\prime}, t\right)\right|^{p} \frac{d z^{\prime}}{\left|y^{\prime}-z^{\prime}\right|^{n+p-2}}+\epsilon_{j}^{\prime \prime \prime},
\end{aligned}
$$

where $\epsilon_{j}^{\prime \prime \prime} \rightarrow 0$ as $j \rightarrow \infty$. From the last estimate and analogous estimates for $B_{j}^{(1)}, B_{j}^{(3)}$ we obtain (5.21).

We now observe that the equality

$$
\left\langle A u_{j}, u_{j}-u_{0}\right\rangle=E_{j}+B_{j},
$$


the estimates (5.19), (5.21), and the condition (5.12) imply

$$
\begin{aligned}
\limsup _{j \rightarrow \infty}\left\{\| \frac{\partial}{\partial t}\left(u_{j}-u_{0}\right)-\right. & L\left(u_{0}\right)\left(u_{j}-u_{0}\right) \|_{p, Q_{T}}^{(2)} \\
& \left.+\left\|B\left(u_{0}\right)\left(u_{j}-u_{0}\right)\right\|_{p, S_{T}}^{\left(3-\frac{1}{p}\right)}\right\}=0 .
\end{aligned}
$$

Applying the a priori estimate (4.8) to the operators $L\left(u_{0}\right), B\left(u_{0}\right)$ and the function $u_{j}-u_{0}$, we obtain

$$
\begin{aligned}
\left\|u_{j}-u_{0}\right\|_{p, Q_{T}}^{(4)} \leq \mathcal{K}\left\{\| \frac{\partial}{\partial t}\left(u_{j}-u_{0}\right)-\right. & L\left(u_{0}\right)\left(u_{j}-u_{0}\right) \|_{p, Q_{T}}^{(2)} \\
& \left.+\left\|B\left(u_{0}\right)\left(u_{j}-u_{0}\right)\right\|_{p, S_{T}}^{\left(3-\frac{1}{p}\right)}\right\}
\end{aligned}
$$

with constant $\mathcal{K}$ independent of $j$. The strong convergence of the sequence $u_{j}$ to $u_{0}$ in $W_{p}^{(4), 0}\left(Q_{T}\right)$ follows now from (5.28) and (5.29). The proof of Theorem 3.1 is complete.

Proof of Theorem 3.2. From the definition of the operators $A_{E}$ and $A_{B}$ it follows immediately that $A_{E}(u, 0)=0$ and $A_{B}(u, 0)=0$ for any function $u \in W_{p}^{(4), 0}\left(Q_{T}\right)$. From (3.2), (3.3) and (3.18) we obtain that the solution of the problem (3.2), (3.3) in $W_{p}^{(4), 0}\left(Q_{T}\right)$ is the solution of the operator equation $A u=0$.

Let $u_{0} \in W_{p}^{(4), 0}\left(Q_{T}\right)$ be a solution of the operator equation $A u=0$. Then from Corollary 3.1 we obtain $f_{0} \in W_{p}^{(2), 0}\left(Q_{T}\right)$ and $g_{0} \in W_{p}^{\left(3-\frac{1}{p}\right), 0}\left(S_{T}\right)$, where

$$
f_{0}(x, t)=\frac{\partial u_{0}}{\partial t}-F\left(x, t, u_{0}, \mathcal{D}^{1} u_{0}, \mathcal{D}^{2} u_{0}\right)-f(x, t)
$$

and

$$
g_{0}(x, t)=G\left(x, t, u_{0}, \mathcal{D}^{1} u_{0}\right)-g(x, t) .
$$

From Theorem 4.1 follows the existence of the solution $v_{0} \in W_{p}^{(4), 0}\left(Q_{T}\right)$ of the problem

$$
\begin{gathered}
\frac{\partial v_{0}(x, t)}{\partial t}-L\left(u_{0}\right) v_{0}(x, t)=f_{0}(x, t), \quad(x, t) \in Q_{T}, \\
B\left(u_{0}\right) v_{0}(x, t)=g_{0}(x, t), \quad(x, t) \in S_{T} .
\end{gathered}
$$

For such a function $v_{0}(x, t)$ we have $\left\langle A u_{0}, v_{0}\right\rangle=0$. Consequently, from (3.11), (3.13)-(3.16) and (3.18) we obtain

$$
0=\left\langle A_{E}\left(u_{0}, f_{0}\right), v_{0}\right\rangle+\left\langle A_{B}\left(u_{0}, g_{0}\right), v_{0}\right\rangle=\left(\left\|f_{0}\right\|_{p, Q_{T}}^{(2)}\right)^{p}+\left(\left\|g_{0}\right\|_{p, S_{T}}^{\left(3-\frac{1}{p}\right)}\right)^{p} .
$$

Therefore, we have $f_{0}(x, t) \equiv 0, g_{0}(x, t) \equiv 0$. Now (5.30) and (5.31) imply that the function $u_{0}(x, t)$ is the solution of the initial-boundary value problem (3.2), (3.3). This finishes the proof of Theorem 3.2. 


\section{SOlVABILITY OF NONLINEAR PARABOLIC PROBLEMS}

We start with the proof of the uniqueness theorem and the proof of the local solvability of the problem (1.1)-(1.3).

Proof of Theorem 3.3. The proof is by contradiction. We assume that $u_{0}(x, t)$ and $u_{1}(x, t)$ are two solutions of the problem (3.2), (3.3) with $u_{0} \neq u_{1}$. Then the function $u(x, t)=u_{1}(x, t)-u_{0}(x, t)$ is the solution of the linear parabolic problem (4.1), (4.2) with $f(x, t) \equiv 0, g(x, t) \equiv 0$ and

$$
\begin{aligned}
& a_{\alpha}(x, t)=\int_{0}^{1} F_{\alpha}\left(x, t, u_{s}, \mathcal{D}^{1} u_{s}, \mathcal{D}^{2} u_{s}\right) d s \\
& b_{\beta}(x, t)=\int_{0}^{1} G_{\beta}\left(x, t, u_{s}, \mathcal{D}^{1} u_{s}\right) d s
\end{aligned}
$$

where $u_{s}=u_{s}(x, t)=s u_{1}(x, t)+(1-s) u_{0}(x, t)$ and $F_{\alpha}(x, t, \xi), G_{\beta}(x, t, \zeta)$ are defined in Conditions $\left.\left.F_{2}\right), G_{2}\right)$. The coefficients $a_{\alpha}(x, t), b_{\beta}(x, t)$ satisfy Conditions $\left.\left.\left.\left.a_{1}\right), a_{2}\right), b_{1}\right), b_{2}\right)$ of Section 4 . We thus obtain $u(x, t) \equiv 0$ by Theorem 4.1 . The uniqueness of the solution of the initial-boundary value problem (3.2), (3.3) is now proved.

Proof of Theorem 3.5. From the reduction of the problem (1.1)-(1.3) to the problem with zero initial conditions it follows that it is sufficient to prove the local solvability of the problem (3.2), (3.3). For a given number $\mathcal{K}$ we shall prove the existence of numbers $R_{0}$ and $T_{0}$, depending on $\mathcal{K}$, such that the inequalities

$$
\|f\|_{p, Q_{T}}^{(2)} \leq \mathcal{K}, \quad\|g\|_{p, S_{T}}^{\left(3-\frac{1}{p}\right)} \leq \mathcal{K}
$$

for $f \in W_{p}^{(2), 0}\left(Q_{T}\right), g \in W_{p}^{\left(3-\frac{1}{p}\right), 0}\left(S_{T}\right)$, imply the following estimate:

$$
\langle A u, u\rangle \geq 0, \quad \text { for } u \in W_{p}^{(4), 0}\left(Q_{T}\right),\|u\|_{p, Q_{T}}^{(4)}=R_{0},
$$

where $T$ is an arbitrary number from the interval $\left(0, T_{0}\right]$ and the operator $A$ is defined by (3.18).

Let $\epsilon, R$ be arbitrary positive numbers. Using the estimates (4.31)-(4.33), it is possible to choose a positive number $T(R, \epsilon)$ such that, for

$$
0<T<T(R, \epsilon), u \in W_{p}^{(4), 0}\left(Q_{T}\right),\|u\|_{p, Q_{T}}^{(4)} \leq R
$$

we have

$$
\begin{gathered}
\sum_{2 s+|\gamma| \leq 2}\left\|\left(\frac{\partial}{\partial t}\right)^{s} \mathcal{D}^{\gamma} u\right\|_{Q_{T}}^{(\delta)} \leq \epsilon, \quad \text { for } 0<\delta<2-\frac{n+2}{p} \\
\sum_{2 s+|\gamma|=3}\left\|\left(\frac{\partial}{\partial t}\right)^{s} \mathcal{D}^{\gamma} u\right\|_{2 p, Q_{T}} \leq \epsilon
\end{gathered}
$$

For $\epsilon \leq 1$ and by using the above inequalities we get

$$
\begin{gathered}
\|L(u) u\|_{p, Q_{T}}^{(2)} \leq \mathcal{C}^{(1)}\|u\|_{p, Q_{T}}^{(4)}, \\
\left\|F\left(\cdot, \cdot, u, \mathcal{D}^{1} u, \mathcal{D}^{2} u\right)\right\|_{p, Q_{T}}^{(2)} \leq \mathcal{C}^{(1)}\|u\|_{p, Q_{T}}^{(4)}
\end{gathered}
$$

for $u, T$ satisfying the conditions (6.4), where the positive constant $\mathcal{C}^{(1)}$ is independent of $u, R, \epsilon, T$. The proof of the estimates (6.7) is analogous to the proof of the estimates (3.7), (3.10). 
In a similar manner it is possible to prove the following:

$$
\begin{gathered}
\|L(u) u-L(0) u\|_{p, Q_{T}}^{(2)} \leq \mathcal{C}^{\prime}(\epsilon)\|u\|_{p, Q_{T}}^{(4)}, \\
\left\|F\left(\cdot, \cdot, u, \mathcal{D}^{1} u, \mathcal{D}^{2} u\right)-L(0) u\right\|_{p, Q_{T}}^{(2)} \leq \mathcal{C}^{\prime}(\epsilon)\|u\|_{p, Q_{T}}^{(4)},
\end{gathered}
$$

for $u(x, t), R$ satisfying the conditions (6.4), where $L(0)$ is the operator defined by (3.5) for $u=0$ and $C^{\prime}(\epsilon)$ tends to zero as $\epsilon$ tends to 0 .

We set

$$
\widetilde{F}(u)(x, t)=F\left(x, t, u(x, t), \mathcal{D}^{1} u(x, t), \mathcal{D}^{2} u(x, t)\right),
$$

for $u \in W_{p}^{(4), 0}\left(Q_{T}\right)$, and evaluate the difference

$$
d_{1} \equiv\left\langle A_{E}\left(u, \frac{\partial u}{\partial t}-\widetilde{F}(u)-f\right), u\right\rangle-\left\langle A_{E}\left(0, \frac{\partial u}{\partial t}-L(0) u\right), u\right\rangle
$$

We have

$$
\begin{aligned}
\left|d_{1}\right| \leq & \left|\left\langle A_{E}\left(u, \frac{\partial u}{\partial t}-\widetilde{F}(u)-f\right)-A_{E}\left(0, \frac{\partial u}{\partial t}-\widetilde{F}(u)-f\right), u\right\rangle\right| \\
& +\left|\left\langle A_{E}\left(0, \frac{\partial u}{\partial t}-\widetilde{F}(u)-f\right\rangle-A_{E}\left(0, \frac{\partial u}{\partial t}-\widetilde{F}(u)\right), u\right\rangle\right| \\
& +\left|\left\langle A_{E}\left(0, \frac{\partial u}{\partial t}-\widetilde{F}(u)\right)-A_{E}\left(0, \frac{\partial u}{\partial t}-L(0) u\right), u\right\rangle\right| .
\end{aligned}
$$

Evaluating the summands on the right-hand side above, and using the estimates (6.7) and (6.8), we obtain

$$
\left|d_{1}\right| \leq \mathcal{C}^{(2)}\left\{\left[R^{p-1}+\mathcal{K}^{p-1}\right] \mathcal{C}^{\prime}(\epsilon) R+\left[R^{p-2}+\mathcal{K}^{p-2}\right] \mathcal{K} R\right\},
$$

with the positive constant $\mathcal{C}^{(2)}$ independent of $u, R, \epsilon, \mathcal{K}, T$, for $u, T$ satisfying (6.4). The constant $\mathcal{C}^{\prime}(\epsilon)$ is the same as in (6.8).

We can also derive an analogous estimate for

$$
d_{2} \equiv\left\langle A_{B}(u, \widetilde{G}(u)-g), u\right\rangle-\left\langle A_{B}(0, B(0) u, u\rangle,\right.
$$

where $\widetilde{G}(u)=G\left(x, t, u(x, t), \mathcal{D}^{1} u(x, t)\right)$ and the operator $B(0)$ is defined by (3.6) with $u=0$. First we establish the estimates

$$
\begin{gathered}
\|B(u)\|_{p, S_{T}}^{\left(3-\frac{1}{p}\right)} \leq \mathcal{C}^{(3)}\|u\|_{p, S_{T}}^{\left(4-\frac{1}{p}\right)}\|\widetilde{G}(u)\|_{p, S_{T}}^{\left(3-\frac{1}{p}\right)} \leq \mathcal{C}^{(4)}\|u\|_{p, S_{T}}^{\left(4-\frac{1}{p}\right)}, \\
\|B(u) u-B(0) u\|_{p, S_{T}}^{\left(3-\frac{1}{p}\right)} \leq \mathcal{C}^{\prime \prime}(\epsilon) \|_{p, S_{T}}^{\left(4-\frac{1}{p}\right)} \\
\|\widetilde{G}(u)-B(0) u\|_{p, S_{T}}^{\left(3-\frac{1}{p}\right)} \leq \mathcal{C}^{\prime \prime}(\epsilon)\|u\|_{p, S_{T}}^{\left(4-\frac{1}{p}\right)}
\end{gathered}
$$

for $u, T$ satisfying conditions (6.4). In $(6.12), \mathcal{C}^{(3)}, \mathcal{C}^{(4)}$ are positive constants independent of $u, R, \epsilon, T$, and the positive constant $\mathcal{C}^{\prime \prime}(\epsilon)$ tends to zero as $\epsilon \rightarrow 0$.

Using (6.12) we obtain the estimate

$$
\begin{aligned}
&\left|d_{2}\right| \leq \mathcal{C}^{(5)}\left\{\mathcal{C}^{\prime \prime}(\epsilon)\|u\|_{p, S_{T}}^{\left(4-\frac{1}{p}\right)}\left[\|u\|_{p, S_{T}}^{\left(4-\frac{1}{p}\right)}+\mathcal{K}\right]^{p-1}\right. \\
&\left.+\mathcal{K}\|u\|_{p, S_{T}}^{\left(4-\frac{1}{p}\right)}\left[\|u\|_{p, S_{T}}^{\left(4-\frac{1}{p}\right)}+\mathcal{K}\right]^{p-2}\right\}
\end{aligned}
$$

where $d_{2}$ is defined in (6.11) and $u, T$ satisfy the conditions (6.4). In (6.13), $\mathcal{C}^{\prime \prime}(\epsilon)$ is the constant from (6.12), while the constant $\mathcal{C}^{(5)}$ is independent of $u, R, \epsilon, \mathcal{K}, T$. 
We estimate the norm of $u$ on the right-hand side of (6.13) by using Lemma 2.2 and (4.30). We assume, in addition, that

$$
T<d^{2},
$$

where $d$ was defined in Section 3 in the introduction of local coordinates. Thus, for $u, T$ satisfying the conditions (6.4), (6.14) we obtain the estimate

$$
\left|d_{2}\right| \leq \mathcal{C}^{(6)}[R+\mathcal{K}]^{p-1}\left(\mathcal{C}^{\prime \prime}(\epsilon) R+\mathcal{K}\right)
$$

From the definition of the operator $A$ in (3.18), the numbers $d_{1}, d_{2}$ in (6.9), (6.11) and the estimates $(6.10),(6.15)$ we obtain the inequality

$$
\begin{aligned}
\langle A u, u\rangle \geq & \left\langle A_{E}\left(0, \frac{\partial u}{\partial t}-L(0) u\right), u\right\rangle+\left\langle A_{B}(0, B(0) u), u\right\rangle \\
& -\mathcal{C}^{(7)}[R+\mathcal{K}]^{p-1}\left\{\left[\mathcal{C}^{\prime}(\epsilon)+\mathcal{C}^{\prime \prime}(\epsilon)\right] R+\mathcal{K}\right\},
\end{aligned}
$$

where $u, T$ satisfy the conditions (6.4), (6.14).

We estimate the sum of the two first summands on the right-hand side of (6.16) by Theorem 4.1. We obtain, from (3.11), (3.13) and (4.8),

$$
\begin{aligned}
\left\langle A_{E}\left(0, \frac{\partial u}{\partial t}-L(0) u\right), u\right\rangle & +\left\langle A_{B}(0, B(0) u), u\right\rangle \\
& =\left[\left\|\frac{\partial u}{\partial t}-L(0) u\right\|_{p, Q_{T}}^{(2)}\right]^{p}+\left[\|B(0) u\|_{p, S_{T}}^{\left(3-\frac{1}{p}\right)}\right]^{p} \\
& \geq \mathcal{C}_{0}\left[\|u\|_{p, Q_{T}}^{(4)}\right]^{p},
\end{aligned}
$$

for $u \in W_{p}^{(4), 0}\left(Q_{T}\right)$ with a positive constant $\mathcal{C}_{0}$ independent of $u, T$.

From (6.16), (6.17) we obtain the estimate

$$
\langle A u, u\rangle \geq\left\{\mathcal{C}_{0}-\mathcal{C}^{(8)}\left[\mathcal{C}^{\prime}(\epsilon)+\mathcal{C}^{\prime \prime}(\epsilon)\right]\right\} R^{p}-\mathcal{C}^{(8)}[R+\mathcal{K}]^{p-1} \mathcal{K},
$$

for $\|u\|_{p, Q_{T}}^{(4)}=R$ and $u, T$ satisfying (6.4), (6.14). In (6.18), the constant $\mathcal{C}^{(8)}$ is independent of $u, R, \epsilon, T$. The above estimate guarantees the ability to choose numbers $R_{0}, T_{0}$ so that (6.3) holds.

We conclude that the estimate (6.3) has been established and that the existence of the solution of the operator equation $A u=0$ satisfying the condition $\|u\|_{p, Q_{T}}^{(4)} \leq$ $R_{0}$ follows from well known results of the theory of generalized monotone operators (see, e.g., [14, Chapter 2, Corollary 4.1 and Theorem 4.4]. Using Theorem 3.2, we complete the proof of Theorem 3.5.

Proof of Theorem 3.6. Taking into consideration the fact that for every $\tau \in[0,1]$ the functions $F_{\tau}(x, t, \xi)$ and $G_{\tau}(x, t, \zeta)$ defined by (3.25) satisfy Conditions $\left.F_{2}\right)-$ $\left.\left.\left.-F_{4}\right), G_{2}\right)-G_{4}\right)$, respectively, we can reduce the problem $(3.26),(3.27)$ to the operator equation

$$
A_{\tau} u=0, \quad u \in W_{p}^{(4), 0}\left(Q_{T}\right),
$$

where the operator $A_{\tau}$ is defined by (3.18), if in the construction of Section 3 we replace the functions $F(x, t, \xi), G(x, t, \zeta)$ by the functions $F_{\tau}(x, t, \xi), G_{\tau}(x, t, \zeta)$, respectively.

By Theorem 3.2 the solvability of the boundary value problem (3.2), (3.3) is equivalent to the solvability of the equation $A_{1} u=0$. According to Theorem 3.1, 
for each $\tau \in[0,1]$ the operator $A_{\tau}$ is bounded and continuous and satisfies Condition $(S)_{+}$on $W_{p}^{(4), 0}\left(Q_{T}\right)$. We introduce a domain $\mathcal{D}_{R}$ in $W_{p}^{(4), 0}\left(Q_{T}\right)$ by

$$
\mathcal{D}_{R}=\left\{u \in W_{p}^{(4), 0}\left(Q_{T}\right):\|u\|_{p, Q_{T}}^{(4)}<R+1\right\},
$$

where $R$ is the number from (3.28). The estimate (3.28) guarantees that for every $\tau \in[0,1]$ we have

$$
A_{\tau} u \neq 0, \quad \text { for } u \in \partial \mathcal{D}_{R} .
$$

Thus, by Theorem 3.4, the degree function $\operatorname{Deg}\left(A_{\tau}, \overline{\mathcal{D}_{R}}, 0\right)$ is well defined for all $\tau \in[0,1]$.

As in the proof of Theorem 3.1, it is possible to prove the following properties of the operators $A_{\tau}$ :

1) For any sequences $u_{j} \in W_{p}^{(4), 0}\left(Q_{T}\right), \tau_{j} \in[0,1]$, such that $\tau_{j} \rightarrow \tau_{0}, u_{j} \rightarrow u_{0}$, we have $A_{\tau_{j}} u_{j} \rightarrow A_{\tau_{0}} u_{0}$;

2) For any sequences $u_{j} \in W_{p}^{(4), 0}\left(Q_{T}\right), \tau_{j} \in[0,1]$ satisfying

$$
u_{j} \rightarrow u_{0}, \quad \lim _{j \rightarrow \infty}\left\langle A_{\tau_{j}} u_{j}, u_{j}-u_{0}\right\rangle=0,
$$

we have $u_{j} \rightarrow u_{0}$.

Thus, by [14, Chapter 2, Theorem 4.1], we have

$$
\operatorname{Deg}\left(A_{0}, \overline{\mathcal{D}_{R}}, 0\right)=\operatorname{Deg}\left(A_{1}, \overline{\mathcal{D}_{R}}, 0\right) .
$$

By the principle of nonzero degree [14, Chapter 2, Corollary 4.1], the solvability of the operator equation $A_{1} u=0$ in $\mathcal{D}_{R}$ follows from the relation

$$
\operatorname{Deg}\left(A_{1}, \overline{\mathcal{D}_{R}}, 0\right) \neq 0 \text {. }
$$

Therefore, we can reduce the proof of the theorem to the proof of (6.23). Recalling (6.22), it is sufficient to check only the relation

$$
\operatorname{Deg}\left(A_{0}, \overline{\mathcal{D}_{R}}, 0\right) \neq 0 \text {. }
$$

For a given positive number $\epsilon$, it is possible to choose a positive number $R(\epsilon)$ such that if

$$
u \in W_{p}^{(4), 0}\left(Q_{T}\right), \quad\|u\|_{p, Q_{T}}^{(4)} \leq R(\epsilon),
$$

then the inequalities (6.5) and (6.6) hold. Then, repeating the proof of (6.3), we can verify that for $u$ satisfying the condition (6.25) we have

$$
\begin{aligned}
\left\langle A_{0} u, u\right\rangle \geq & \left.\left\langle A_{E}^{(0)}\left(0, \frac{\partial u}{\partial t}-L_{0}(0) u\right), u\right)\right\rangle+\left\langle A_{B}^{(0)}\left(0, B_{0}(0) u, u\right\rangle\right. \\
& -\mathcal{C}^{(8)}[R(\epsilon)]^{p} \cdot \mathcal{C}^{\prime \prime \prime}(\epsilon),
\end{aligned}
$$

where $\mathcal{C}^{\prime \prime \prime}(\epsilon) \rightarrow 0$ as $\epsilon \rightarrow 0$. Here, the operators $A_{E}^{(0)}, L_{0}, A_{B}^{(0)}, B_{0}$ are defined by (3.5), (3.6), (3.11), (3.13) with the functions $F(x, t, \xi), G(x, t, \zeta)$ replaced by $F_{0}(x, t, \xi), G_{0}(x, t, \zeta)$, respectively.

Estimating the first two summands on the right-hand side of (6.26), obtaining estimates analogous to those of (6.17), we see that we can choose a positive number $\epsilon_{0}$ such that

$$
\left\langle A_{0} u, u\right\rangle>0 \quad \text { for }\|u\|_{p, Q_{T}}^{(4)}=R\left(\epsilon_{0}\right) .
$$


By [14, Chapter 2, Theorem 4.4], we obtain from (6.27)

$$
\operatorname{Deg}\left(A_{0}, \overline{\mathcal{D}\left(\epsilon_{0}\right)}, 0\right)=1,
$$

where

$$
\mathcal{D}\left(\epsilon_{0}\right)=\left\{u \in W_{p}^{(4), 0}\left(Q_{T}\right):\|u\|_{p, Q_{T}}^{(4)}<R\left(\epsilon_{0}\right)\right\} .
$$

From the uniqueness of the solution of the equation $A_{0} u=0$ (Theorem 3.3), 14 Chapter 2, Theorem 5.1] and (6.28) we obtain $\operatorname{Deg}\left(A_{0}, \overline{\mathcal{D}_{R}}, 0\right)=1$. Thus, we have shown the inequality (6.24), and the proof is finished.

Remark 6.1. Using a proof similar to that of (6.28), it is possible to establish the following property of the degree function for the operator $A$ defined by (3.18). Let $\mathcal{D}$ be a bounded open set in $W_{p}^{(4), 0}\left(Q_{T}\right)$ such that $A u \neq 0, u \in \partial \mathcal{D}$. Then

$$
\operatorname{Deg}(A, \overline{\mathcal{D}}, 0)=1
$$

if and only if there exists a solution $u$ of the equation $A u=0$ in $\mathcal{D}$. Otherwise $\operatorname{Deg}(A, \overline{\mathcal{D}}, 0)=0$.

Finally, we formulate an invariance of domain result for the problem (3.2), (3.3). An analogous result can be proved for the problem (1.1)-(1.3).

Theorem 6.1. Assume that the conditions of Theorem 3.1 are satisfied and let $\mathcal{D}$ be an open set in $W_{p}^{(4), 0}\left(Q_{T}\right)$. Then the set

$$
R(\mathcal{D})=\left\{\left(\frac{\partial u}{\partial t}-F\left(\cdot, \cdot, u, \mathcal{D}^{1} u, \mathcal{D}^{2} u\right), G\left(\cdot, \cdot, u, \mathcal{D}^{1} u\right)\right): u \in \mathcal{D}\right\}
$$

is open in $W_{p}^{(2), 0}\left(Q_{T}\right) \times W_{p}^{\left(3-\frac{1}{p}\right), 0}\left(S_{T}\right)$.

Proof. Let $u_{0} \in \mathcal{D}$ be given and let

$$
f_{0}(x, t)=\frac{\partial u_{0}}{\partial t}-F\left(x, t, u_{0}, \mathcal{D}^{1} u_{0}, \mathcal{D}^{2} u_{0}\right), \quad g_{0}(x, t)=G\left(x, t, u_{0}, \mathcal{D}^{2} u_{0}\right) .
$$

We have to show that there exists a positive number $\rho$ such that for every pair of functions $f \in W_{p}^{(2), 0}\left(Q_{T}\right), g \in W_{p}^{\left(3-\frac{1}{p}\right), 0}\left(S_{T}\right)$ satisfying the inequalities

$$
\left\|f-f_{0}\right\|_{p, Q_{T}}^{(2)}<\rho, \quad\left\|g-g_{0}\right\|_{p, S_{T}}^{\left(3-\frac{1}{p}\right)}<\rho
$$

the boundary value problem (3.2), (3.3) has a solution $u \in \mathcal{D}$. We may choose a positive number $\epsilon$ such that

$$
\overline{B\left(u_{0}, \epsilon\right)} \subset \mathcal{D} .
$$

By the uniqueness Theorem 3.3,

$$
A_{0} u \neq 0 \quad \text { for } u \in \partial B\left(u_{0}, \epsilon\right),
$$

where

$$
\begin{aligned}
A_{0} u= & A_{E}\left(u, \frac{\partial u}{\partial t}-F\left(x, t, u, \mathcal{D}^{1} u, \mathcal{D}^{2} u\right)-f_{0}(x, t)\right) \\
& +A_{B}\left(u, G\left(x, t, u, \mathcal{D}^{2} u\right)-g_{0}(x, t)\right) .
\end{aligned}
$$

Consequently, by Remark 6.1, we have

$$
\operatorname{Deg}\left(A_{0}, \overline{B\left(u_{0}, \epsilon\right)}, 0\right)=1 \text {. }
$$


Using (6.31), Condition $(S)_{+}$and the continuity of the operator $A_{0}$, it is easy to check that

$$
\left\|A_{0} u\right\|_{*} \geq \eta \quad \text { for } u \in \partial B\left(u_{0}, \epsilon\right),
$$

with some positive number $\eta$, where $\|\cdot\|_{*}$ is the norm in the space $\left[W_{p}^{(4), 0}\left(Q_{T}\right)\right]^{*}$. We can now choose the number $\rho$ such that for $f \in W_{p}^{(2), 0}\left(Q_{T}\right), g \in W_{p}^{\left(3-\frac{1}{p}\right), 0}\left(S_{T}\right)$ satisfying (6.29) we have

$$
\left\|A u-A_{0} u\right\|_{*} \leq \frac{\eta}{2} \text { for } u \in \partial B\left(u_{0}, \epsilon\right),
$$

where the operator $A$ is defined by (3.18).

We now consider a one-parameter family of linear mappings

$$
A_{\tau}: \overline{B\left(u_{0}, \epsilon\right)} \rightarrow\left[W_{p}^{(4), 0}\left(Q_{T}\right)\right]^{*}, \quad A_{\tau}=(1-\tau) A_{0} u+\tau A u,
$$

for $\tau \in[0,1]$. Using (6.34) and (6.35), it is easy to verify that this is a homotopy family for the operators $A_{0}, A$ on $\overline{B\left(u_{0}, \epsilon\right)}$. Thus, by [14, Chapter 2, Theorem 4.1] and (6.33) we get

$$
\operatorname{Deg}\left(A, \overline{B\left(u_{0}, \epsilon\right)}, 0\right)=1 .
$$

From this and the principle of nonzero degree in [14, Chapter 2, Corollary 4.1] follows the existence of the solution of the equation $A u=0$ in $B\left(u_{0}, \epsilon\right)$. By Theorem 3.2 we have the solution $u \in \mathcal{D}$ of the problem (3.2), (3.3) for an arbitrary pair of functions $f, g$ satisfying condition (6.29). This is the end of the proof.

The next corollary follows from Theorem 6.1 and for $\mathcal{D}=W_{p}^{(4), 0}\left(Q_{T}\right)$.

Corollary 6.1. The set of pairs

$$
\begin{array}{r}
R^{(0)}=\left\{(f, g) \in W_{p}^{(2), 0}\left(Q_{T}\right) \times W_{p}^{\left(3-\frac{1}{p}\right), 0}\left(S_{T}\right)\right. \text { : the boundary value problem } \\
\text { (3.2), (3.3) has a solution in } \left.W_{p}^{(4), 0}\left(Q_{T}\right)\right\}
\end{array}
$$

is open in $W_{p}^{(2), 0}\left(Q_{T}\right) \times W_{p}^{\left(3-\frac{1}{p}\right), 0}\left(S_{T}\right)$.

Corollary 6.2. Let $U: R^{(0)} \rightarrow W_{p}^{(4), 0}\left(Q_{T}\right)$ be the operator which maps the pair $(f, g) \in R^{(0)} \quad\left(R^{(0)}\right.$ is the set defined in Corollary 6.1) to the solution $u$ of the problem (3.2), (3.3) (i.e., $U(f, g)=u$ ). Then the operator $U$ is continuous.

Corollary 6.2 follows from the proof of Theorem 6.1.

\section{Convergence of the Galerkin approximants}

We let $v_{i}, i=1,2, \ldots$, be a complete system of functions in the space $W_{p}^{(4), 0}\left(Q_{T}\right)$ such that for any index $I$ the functions $v_{1}, v_{2}, \ldots, v_{I}$ are linearly independent.

By a " $j$-approximate solution" of the initial-boundary value problem (3.2), (3.3) we mean a function $u_{j}$ such that

$$
u_{j}(x, t)=c_{1}^{(j)} v_{1}(x, t)+\cdots+c_{j}^{(j)} v_{j}(x, t)
$$


and

$$
\begin{aligned}
\left\langleA _ { E } \left( u_{j}, \frac{\partial u_{j}}{\partial t}\right.\right. & \left.\left.\left.-F\left(\cdot, \cdot, u_{j}, \mathcal{D}^{1} u_{j}, \mathcal{D}^{2} u_{j}\right)-f\right)\right), v_{i}\right\rangle \\
& \left.+\left\langle A_{B}\left(u_{j}, G\left(\cdot, \cdot, u_{j}, \mathcal{D}^{2} u\right)-g\right)\right), v_{i}\right\rangle=0, \quad i=1, \ldots, j,
\end{aligned}
$$

where the operators $A_{E}, A_{B}$ are defined by (3.11), (3.13), respectively, and the $c_{i}^{(j)}$ in (7.1) are real numbers.

We say that the problem (3.2), (3.3) has a "bounded sequence of approximate solutions" if there exists a number $j_{0}$ such that for each $j \geq j_{0}$ the problem (3.2), (3.3) has a $j$-approximate solution $u_{j}(x, t)$ and the sequence $\left\{u_{j}\right\}, j=j_{0}, j_{0}+$ $1, \ldots$, is bounded in $W_{p}^{(4), 0}\left(Q_{T}\right)$.

Lemma 7.1. Assume that Conditions (2.11), (3.4), $\left.\left.\left.F_{2}\right)-F_{4}\right), G_{2}\right)-G_{4}$ ) are satisfied. Then the initial-boundary value problem (3.2), (3.3) has a bounded sequence of approximate solutions if and only if there exists a solution of this problem in the space $W_{p}^{(4), 0}\left(Q_{T}\right)$.

Proof. Let $\left\{u_{j}\right\}$ be a bounded sequence of approximate solutions. We can choose a subsequence $\left\{u_{j(k)}\right\}, k=1,2, \ldots$, which converges weakly to some function $u_{0} \in W_{p}^{(4), 0}\left(Q_{T}\right)$.

Denote by $F_{j}$ the linear hull of the functions $v_{1}, \ldots, v_{j}$. By our assumptions on the system $\left\{v_{j}\right\}$, there exists a sequence $\left\{u_{j}^{(0)}\right\}, j=1,2, \ldots$, such that $u_{j}^{(0)} \in F_{j}$ and $u_{j}^{(0)}$ converges strongly in $W_{p}^{(4), 0}\left(Q_{T}\right)$ to $u_{0}$.

By the definition of the $j$-approximate solution we have

$$
\left\langle A u_{j(k)}, u_{j(k)}-u_{0}\right\rangle=\left\langle A u_{j(k)}, u_{j(k)}^{(0)}-u_{0}\right\rangle
$$

with the operator $A$ defined by (3.18). The right-hand side of (7.3) tends to zero as $k \rightarrow \infty$ because $A$ is a bounded operator. Using Theorem 3.1 and Condition $(S)_{+}$, we establish the strong convergence of the sequence $\left\{u_{j(k)}\right\}$ to $u_{0}$. Passing to the limit in

$$
\left\langle A u_{j(k)}, v_{i}\right\rangle=0
$$

as $k \rightarrow \infty$, we obtain, by the continuity of $A$, that $\left\langle A u_{0}, v_{i}\right\rangle=0$ for any $i$. Since the system $\left\{v_{i}\right\}$ is complete, we have $A u_{0}=0$ and, consequently, $u_{0}$ is the solution of the problem (3.2), (3.3) by Theorem 3.2.

Let $\tilde{u}(x, t)$ be a solution of the problem (3.2), (3.3). We shall prove the existence of a bounded sequence of approximate solutions. Set $R=\|\tilde{u}\|_{p, Q_{T}}^{(4)}$ and

$$
\mathcal{D}_{R}=\left\{u \in W_{p}^{(4), 0}\left(Q_{T}\right):\|u\|_{p, Q_{T}}^{(4)}<R+1\right\} .
$$

By Theorem 3.3, $A u \neq 0$ for $u \in \partial \mathcal{D}_{R}$. It follows that the degree $\operatorname{Deg}\left(A, \overline{\mathcal{D}_{R}}, 0\right)$ is well defined, and we have

$$
\operatorname{Deg}\left(A, \overline{\mathcal{D}_{R}}, 0\right)=1
$$

by Remark 6.1 .

Define, for $j=1,2, \ldots$, the finite-dimensional mapping $A_{j}$ corresponding to the operator $A$ in the following way:

$$
A_{j} u=\sum_{i=1}^{j}\left\langle A u, v_{i}\right\rangle v_{i}, \quad \text { for } u \in \overline{\mathcal{D}_{R} \cap F_{j}} .
$$


By the definition of the degree $\operatorname{Deg}\left(A, \overline{\mathcal{D}_{R}}, 0\right)$ (see [13, Chapter 2, Paragraph 2]), the finite-dimensional degree $\operatorname{deg}\left(A_{j}, \overline{\mathcal{D}_{R} \cap F_{j}}, 0\right)$ is well defined for all large $j$ and

$$
\lim _{j \rightarrow \infty} \operatorname{deg}\left(A_{j}, \overline{\mathcal{D}_{R} \cap F_{j}}, 0\right)=\operatorname{Deg}\left(A, \overline{\mathcal{D}_{R}}, 0\right)=1 .
$$

By the solvability property of the finite-dimensional degree we obtain from (7.6) the existence of a sequence $\left\{u_{j}\right\}, j \geq j_{0}$, for some $j_{0}$, such that

$$
u_{j} \in \mathcal{D}_{R} \cap F_{j} \quad \text { and } \quad A_{j} u_{j}=0 .
$$

The equality (7.7) means that $u_{j}$ is a $j$-approximate solution of the problem (3.2), (3.3). For this function $u_{j}$ we have the estimate $\left\|u_{j}\right\|_{p, Q_{T}}^{(4)}<R+1$ and, consequently, $\left\{u_{j}\right\}$ is a bounded sequence of approximate solutions. This completes the proof of Lemma 7.1.

Theorem 7.1. Assume that Conditions (2.11), (3.4), $\left.\left.\left.F_{2}\right)-F_{4}\right), G_{2}\right)-G_{4}$ ) are satisfied and the initial-boundary value problem (3.2), (3.3) has a solution $u_{0} \in$ $W_{p}^{(4), 0}\left(Q_{T}\right)$. Then for all large $j$ there exists a $j$-approximate solution $u_{j}$ of the problem (3.2), (3.3). The sequence $\left\{u_{j}\right\}$ converges strongly to $u_{0}$ in $W_{p}^{(4), 0}\left(Q_{T}\right)$.

Proof. The existence of a bounded sequence of approximate solutions follows immediately from Lemma 7.1. The strong convergence of the sequence $\left\{u_{j}\right\}$ to $u_{0}$ follows from the first part of the proof of Lemma 7.1 and the uniqueness Theorem 3.3.

\section{ACKNOWLEDGMENT}

This paper was written, with the partial support of a COBASE grant, while Professor Igor V. Skrypnik was visiting the University of South Florida, Tampa, Florida, USA, in 1996-97. Professor Skrypnik wishes to express his thanks to Professor A. G. Kartsatos for his hospitality and their interesting collaboration during his stay in Tampa.

Added in Proof. The authors have now developed degree theories for densely defined mappings of a type like $(S+)$, and perturbations of maximal monotone operators by such mappings. For these degree theories, the reader is referred to "Topological Degree Theories for Densely Defined Mappings Involving Operators of Type (S+)", Adv. Differential Equations, 4 (1999), 413-456. For an index of a critical point theory of such topological degrees, we cite the authors' paper "The index of a Critical Point for Densely Defined Operators of Type $(S+)$ in Banach Spaces", to appear. Applications of the index theory to boundary value problems for partial differential equations can be found in the authors' paper "The Index of a Critical Point for Nonlinear Elliptic Operators With Strong Coefficient Growth", J. Math. Soc. Japan, to appear.

\section{REFERENCES}

[1] P. Acquistapace and B. Terreni, Fully nonlinear parabolic systems, Recent Advances in Nonlinear Elliptic and Parabolic Problems (Nancy, 1988), Pitman Res. Notes in Math., vol. 203, Longman Sci. Tech., Harlow, 1989, pp. 97-111. MR 92a:35080

[2] H. Amann, Quasilinear parabolic systems under nonlinear boundary conditions, Arch. Rat. Mech. Anal. 92 (1986), 153-192. MR 87a:35101]

[3] G. C. Dong, Initial and oblique boundary value problems for fully nonlinear parabolic equations, J. Partial Differential Equations 1 (1988), 12-42. MR 90g:35073 
[4] S. I. Hudjaev, The first boundary value problem for nonlinear parabolic equations, Dokl. Akad. Nauk SSSR 149 (1963), 535-538; English transl., Soviet Math. Dokl. 4 (1963), 441445. MR 28:1404

[5] V. P. Il'in, The properties of some classes of differentiable functions of several variables defined in an n-dimensional region, Trudy Mat. Inst. Steklov 66 (1962), 227-363; English transl., Amer. Math. Soc. Transl. (2) 81 (1969), 91-256. MR 27:3750

[6] S. N. Kruzhkov, A. Castro and M. Lopes, Schauder type estimates and theorems on the existence of the solution of fundamental problem for linear and nonlinear parabolic equations, Dokl. Akad. Nauk SSSR 220 (1975), 277-280; English transl., Soviet Math. Dokl. 16 (1975), 60-64. MR 52:14656

[7] N. V. Krylov, Nonlinear elliptic and parabolic equations of the second order, Nauka, Moscow, 1985; English transl., Reidel, Dordrecht, 1987. MR 87h:35002 MR 88d:35005

[8] O. A. Ladyzhenskaya, V. A. Solonnikov and N. N. Uraltseva, Linear and quasilinear equations of parabolic type, Nauka, Moscow, 1967; English transl., Amer. Math. Soc., Providence, RI, 1968. MR 39:3159a: MR 39:3159b

[9] G. M. Lieberman, Second order parabolic differential equations, World Scientific, 1996, Singapore, 1996. MR 98k:35003

[10] A. Lunardi, Maximal space regularity in nonhomogeneous parabolic problems, Numer. Funct. Anal. Optim. 10 (1989), 323-349. MR 90e:35093

[11] A. Lunardi, On a class of fully nonlinear parabolic equations, Comm. Partial Differential Equations, 16 (1991), 145-172. MR 92c:35058

[12] L. Nirenberg, On elliptic partial differential equations, Ann. Scuola Norm. Sup. Pisa, 13 (1959), 115-162. MR 22:823

[13] I. V. Skrypnik, Nonlinear higher order elliptic equations, Naukova Dumka, Kiev, 1973. (Russian) MR 55:8544

[14] I. V. Skrypnik, Methods for analysis of nonlinear elliptic boundary value problems, Transl. Math. Monographs, vol. 139, Amer. Math. Soc., Providence, RI, 1994. MR 95i:35109

[15] V. A. Solonnikov, A priori estimates for second order parabolic equations, Trudy Mat. Inst. Steklov 70 (1964), 133-212; English transl., Amer. Math. Soc. Transl. (2) 65 (1967), 51-137. MR 28:5267.

[16] N. N. Sopolov, The first boundary value problem for nonlinear parabolic equations of any order, C. R. Acad. Bulgare Sci. 23 (1970), 899-902 (Russian). MR 51:1130

[17] N. N. Uraltseva, A nonlinear broblem with an oblique derivative for a parabolic equation, J. Math. Sci. 70 (1994), 1817-1827. MR 92d:35160 (Russian original)

[18] L. Wang, On the regularity theory of fully nonlinear parabolic equations, Comm. Pure Appl. Math. 45 (1992), 27-76, 141-178. MR 92m:35126; MR 92m:35127

Department of Mathematics, University of South Florida, Tampa, Florida 33620-5700 E-mail address: hermes@math.usf.edu

Institute for Applied Mathematics and Mechanics, R. Luxemburg Str. 74, Donetsk 340114, UKRAINE

E-mail address: skrypnik@iamm.ac.donetsk.ua 